\title{
Systematic analysis of multiple voting rules
}

\author{
Donald G. Saari
}

Received: 18 August 2008 / Accepted: 13 January 2009 / Published online: 5 May 2009

(C) The Author(s) 2009. This article is published with open access at Springerlink.com

\begin{abstract}
For a class of voting rules, which includes Approval and Cumulative Voting, it is shown how to find and analyze all possible outcomes that arise with a specified profile, and, conversely, how to start with a potential region and determine whether there exist supporting profiles. The geometry of these regions is determined by the "Reversal symmetry" portion of a profile; i.e., components of the $A \succ B \succ$ $C, C \succ B \succ A$ type.
\end{abstract}

\section{Introduction}

The surprising complexity of voting theory almost forces comparisons and analyses of voting rules to emphasize how they fare only in special cases or with respect to specific properties. Rather than being able to develop a desired general overview, the complicated analysis may limit us, for instance, to focus only on which rules elect a Condorcet or a majority winner when one exists, or satisfy specified assumptions, or provide reasonable outcomes for a particular profile. While these results contribute to our understanding of voting rules, the conclusions tend to be specific to the posed properties and examples. As such, the literature assumes an ad hoc flavor where a conclusion from one analyzed situation need not tell us much about what else can happen, nor even how a conclusion relates to closely related settings. For instance, knowing that a rule always elects a majority winner does not tell us whether it always elects the weaker Condorcet winner.

This research was supported by NSF grants DMI-0640817 and DMS-0631362. My thanks to a referee who gave the manuscript a particularly thorough reading; the suggestions helped a lot!

D. G. Saari (凷

Institute for Mathematical Behavioral Sciences, University of California, Irvine, CA 92697-5100, USA

e-mail: dsaari@uci.edu 
A way to help correct this problem is to create systematic approaches to analyze classes of voting rules; e.g., find appropriate frameworks from which it is possible to obtain answers for several issues and questions. This paper contributes toward this development for the class of multiple rules; these are the voting rules where a voter has two or more ways to tally his or her ballot. [While I emphasize Approval (AV) and Cumulative Voting (CV), my approach applies to all such systems including Range Voting (RV).] A reason these rules prove to be difficult to analyze is that, typically, a profile defines several different election rankings rather than just the normally anticipated unique outcome. These differences in tallies and outcomes represent the voters' behavioral differences as reflected by how they choose to tally their ballots. But behavioral aspects are based on information that typically is not included in a profile; thus there is a need to develop analytic approaches with which we can systematically analyze these systems.

To illustrate what I mean, during the 1993 US Senate confirmation proceedings for Lani Guinier for a position in the US government, a controversy arose over her support of $\mathrm{CV}$ as a voting method to help minorities elect more candidates. It is easy to show that minorities can do better with $\mathrm{CV}$ if they vote in a specific manner and all other voters vote in a different specified way. But what if other behavioral assumptions apply; e.g., what happens if the other voters refuse to vote as postulated? Might CV have unexpected and unintended surprises? How does CV compare with, say, AV in achieving these goals?

To the best of my knowledge, there does not exist a systematic way to answer these kinds of CV questions. In large part, this gap reflects the complexity of analyzing CV and other multiple rules. So, to create a way to address these issues, in this paper, I develop a geometric tool that will identify all possible AV and CV outcomes that can occur with any combination of different voter behaviors for any specified profile. Answers about the consequences of different behavioral assumptions for multiple methods, such as $\mathrm{CV}$, follow from the geometry.

As an example involving $\mathrm{AV}$, we may wish to know for the profile

\begin{tabular}{|c|c|c|c|}
\hline Number & Ranking & Number & Ranking \\
\hline 10 & $A \succ B \succ C$ & 4 & $C \succ B \succ A$ \\
\hline 7 & $A \succ C \succ B$ & 16 & $B \succ C \succ A$ \\
\hline 11 & $C \succ A \succ B$ & 0 & $B \succ A \succ C$ \\
\hline
\end{tabular}

all of the behavioral assumptions that support the AV outcome of $B \succ A \succ C$ with a $(18,29,16)$ tally, or $C \succ B \succ A$ with the $(22,23,26)$ tally, or some other outcome. With the approach developed here, answers are immediate.

\subsection{Multiple voting rules}

A "multiple voting rule" (Saari and Van Newenhizen 1988) is where a voter ranks the candidates and then selects a positional rule to tally the ballot from two or more choices. An $n$-candidate positional voting rule is defined by specified weights $\left(w_{1}, w_{2}, \ldots, w_{n}=0\right)$ where not all weights agree and $w_{j} \geq w_{j+1}$. In tallying a ballot, $w_{j}$ points are assigned to the candidate who is positioned in the $j$ th rank; 
each candidate's societal ranking is determined by the sum of her assigned points. The choices of $(1,0,0),(2,1,0)$, and $(1,1,0)$ represent, respectively, the plurality vote, Borda Count, and vote-for-two rule. As the three candidate "vote-for-two" rule is equivalent to voting against one candidate, it also is called the "antiplurality vote."

Multiple rules are easy to find. Consider, for example, a $(5,2,0)$ positional election where, if a voter truncates his ballot by listing only his top-ranked candidate, she receives five points and the other candidates receive zero. As the voter's actions determine whether to use $(5,2,0)$ or $(5,0,0)$ to tally his ballot, this becomes a multiple voting rule. (In Sect. 2.1.2, all possible outcomes permitted by this rule with a specified profile are computed.)

All multiple rules can be analyzed as described in this article, but AV and CV are emphasized to convey the ideas. These methods are selected because they have received attention both in theory and in actual elections and because they illustrate different features of my geometric constructions. For specificity, I use the following definition of the main rules mentioned in this article:

Definition 1 "Approval Voting" (AV) is where a voter votes "yes" or "no" for each candidate; in tallying the ballot, a candidate receiving a "yes" vote receives one point.

"Cumulative voting" (CV) is where each voter has a fixed integer number of points; the voter assigns integer values to the candidates in any desired manner as long as the sum does not exceed the specified value. With $n$ candidates, assume that the total is $n-1$.

"Divide the points voting" (DPV) is where each voter is given a fixed number of points. The voter assigns non-negative values to the candidates, whether integer valued or not, in any desired manner as long as the sum equals the specified value.

"Range Voting" (RV) is where each voter assigns to each candidate a number of points coming from a specified range, say, any integer between 0 and 100 .

In all cases, each candidate is ranked according to the sum of assigned points.

So, after a voter ranks the three candidates, with AV he selects either the plurality or antiplurality rule, with CV he selects the $(2,0,0)$, the $(1,0,0)$, or the $(1,1,0)$ rule, and with RV he selects one of the over five thousand different positional rules that are defined by the $w_{1} \geq w_{2} \geq 0$ values in the specified range.

To appreciate the added complexity that the behavioral variable imposes on multiple rules, notice that these rules do not satisfy even standard properties such as "anonymity." The choice theory definition of anonymity, of course, has nothing to do with the secret ballot; it reflects the notion that an election outcome depends on voter preferences rather than on which voter has what ranking. Thus, anonymity requires an interchange of preferences among the voters to yield precisely the same outcome. But, illustrating with AV, if Steve always votes for his top two candidates, while Sue always votes for only one, then interchanging their preferences can violate anonymity by changing the election tallies. The next section provides a way to determine what standard properties are satisfied.

\subsection{An analytic method}

A tool to be developed here to systematically analyze multiple rules with three candidates is based on a "profile decomposition" (Saari 1999, 2008). This decomposition, 
which defines a "profile coordinate system," was created to explain all possible ways in which all positional rules over all three candidates and majority votes over pairs can agree or differ. One of the "profile directions," for instance, has no effect on pairwise rankings, but it is responsible for all possible differences among positional rules. This "Reversal symmetry" involves a ranking and its reversal such as

$$
\{A \succ B \succ C, C \succ B \succ A\} .
$$

A typical result is that if (in a linear algebra sense) a profile has no traces of Reversal symmetry, then all positional election outcomes agree. Because multiple methods are defined in terms of positional rules, this symmetry must be expected to play a major role in analyzing and understanding their properties-it does.

A second profile direction, which I call the "Condorcet symmetry," has no influence on positional rankings, but it is responsible for all possible voting problems with pairs. This profile direction is captured by Condorcet triplets such as

$$
A \succ B \succ C, \quad B \succ C \succ A, \quad C \succ A \succ B .
$$

A typical result is that a profile with no traces of this Condorcet symmetry experiences no pairwise inconsistencies; e.g., the Borda and Condorcet winners (and rankings) agree. Conversely, all possible differences between Borda and pairwise rankings can be completely explained in terms of this profile direction.

The remaining "orthogonal" profile directions, called Basic profiles, are stripped of all Reversal and Condorcet symmetry effects. With these profile components, all positional and pairwise outcomes completely agree. (All of these coordinates are defined in Sect. 3.)

Important for our development is the separability of the Eqs. 2 and 3 profile directions; each causes all possible election differences for one class of voting rules, but it has absolutely no influence on the other. To indicate how to use this feature, recall my earlier question about which of the rules that always elects a majority winner will also always elect a Condorcet winner. As the Condorcet winner involves pairwise comparisons, such a rule must be sensitive to profile components in the Condorcet direction. This condition, then, immediately eliminates all methods that are based strictly on positional rules; e.g., while the plurality vote always elects a majority winner, it need not elect a Condorcet winner.

A way to use this profile decomposition to analyze multiple rules is to partition the voters into sets according to their choices of a positional voting rule. In our setting of three candidates, $\mathrm{AV}$, for instance, defines two partition sets: one consists of those voters who use the plurality vote and the other of those who use the antiplurality vote. Next, analyze each partition set with the profile decomposition, and then combine the results from the different partitions in the obvious manner. (An actual "nail biting" AV election was analyzed in this manner in Saari 2001b.)

By using this approach, it becomes clear how classical results, such as Arrow's Theorem, hold for multiple systems; just apply the result to each partition set of voters. To demonstrate, consider the standard issue of determining whether a voting rule 
always elects a Condorcet winner (a candidate who wins all majority vote pairwise comparisons) when one exists. The following assertion, which contradicts statements in the literature [e.g., recall the assertions in Brams and Fishburn (1978) about an AV winner being a Condorcet winner; also see the exchange between Brams et al. (1988) with Saari and Van Newenhizen (1988)], follows immediately by partitioning of voters and using the profile decomposition.

Theorem 1 If any non-Borda positional method is a choice for a multiple rule, then the rule's ranking need not be related, in any way, with how the pairs of candidates are ranked with the majority vote. As special cases for three candidates, each of AV, $C V, D P V$, and RV could have the Condorcet winner bottom, middle, or top ranked; each could elect the Condorcet loser.

Proof To illustrate with AV, select any profile $\mathbf{p}_{1}$ where the majority votes over pairs defines the $A \succ B \succ C$ ranking (i.e., $A$ is the Condorcet winner by beating both $B$ and $C$, and $B$ beats $C$ ), but the plurality ranking is the reversed $C \succ B \succ A$; select another profile $\mathbf{p}_{2}$ where the same outcomes hold for the antiplurality ranking. (The profile decomposition (Saari 1999) shows how to construct all possible examples by using Reversal and Condorcet symmetries.) The illustrating profile is $\mathbf{p}=\mathbf{p}_{1}+\mathbf{p}_{2}$ where the partitioning is as constructed. So $C$, the Condorcet loser, is the AV winner, while $A$, the Condorcet winner, is the AV loser. The same argument holds for CV, DPV, and RV. More generally, select a ranking for any non-Borda rule and another one for the pairs; because, as shown in (Saari 1999), a supporting profile can be found, this result extends to the indicated multiple rules.

To see why Arrow's Theorem Arrow (1963) applies to all multiple voting rules, first notice that no multiple rule is dictatorial, all voters can have transitive preferences without restrictions, and, because the societal outcome is based on numeric scores, all outcomes are transitive. The last of Arrow's conditions to be checked for each rule is whether there exist special situations whereby it violates the Pareto Condition and/or Independence of Irrelevant Alternatives (IIA). But because plurality voting is a special case of $\mathrm{AV}, \mathrm{RV}, \mathrm{DPV}$, and $\mathrm{CV}$, and because the plurality vote violates IIA, it follows that such special situations exist for each multiple rule where it violates IIA. This is what Arrow's theorem asserts.

To show how to design illustrating examples, consider a three candidate RV election. Suppose five voters with $A \succ B \succ C$ preferences select their choices of weights to assign to the top two candidates and zero to the third to obtain the $A: B: C$ tallies of 52:48:0. Suppose the other five voters, with the $B \succ A \succ C$ ranking, select points to assign to their top two candidates yielding the $A: B: C$ tally of 49:50:0. Thus, in the societal ranking $A$ beats $B$ with the 101:98 tally. As IIA requires this $\{A, B\}$ societal ranking to remain fixed for any profile where each voter has the same $\{A, B\}$ ranking, modify the original profile so that the second five voters now have $B \succ C \succ A$ rankings. Although each voter's $\{A, B\}$ relative ranking is unchanged in both profiles, $A$ is bottom ranked for the second five voters, so they do not assign her any points. As such, the outcome has the reversed pairwise $B \succ A$ ranking that violates IIA. 


\subsection{Geometry}

By partitioning the voters into different groups and using the profile decomposition, it is possible to find answers for several multiple voting rule issues. But as this approach requires knowing how to partition the voters into different subsets, many other concerns cannot be tackled in this manner; e.g., we cannot determine consequences that are associated with all possible ways to divide the voters into different partition sets. As such, there is a need to develop tactics with which we can analyze all possible ways in which the "behavioral variable" affects election outcomes.

To accomplish this objective, in Sects. 2-5, I develop a geometric technique to characterize all possible election rankings and tallies that are admitted by a particular multiple rule for any specified profile. The ultimate objective is clear; because the approach identifies everything that can happen, it can be used to determine what kinds of voter divisions cause the different outcomes. Namely, this approach can serve as a tool to analyze how different behavioral assumptions, including strategic voting, affect multiple rule election outcomes.

Somewhat unexpected and as shown in Sect. 3, the results have a pleasing geometric symmetry; e.g., it turns out that all AV outcomes defined by a profile are in a rectangular box that is symmetrically centered about the Borda tally. This symmetric centering property extends to other multiple rules; e.g., all possible CV outcomes lie in a symmetrically shaped polyhedron that is centered about the outcome for a second positional rule, and the box of outcomes associated with the above truncated voting example is centered about a third rule. Moreover, the shape of each box is determined by the profile's Reversal symmetry components, and the boxes for the four multiple rules emphasized here include the profile's election ranking for each possible positional rules. As such, any perceived virtue or fault that is associated with any positional rule becomes a special case for each of the multiple rules.

Because the geometric shape of a multiple rule's box of voting outcomes is determined by a profile's Reversal symmetries, this symmetry is used in Sects. 3 and 4 to create an easier way to determine the set of all possible outcomes and to analyze the behavioral aspects of each multiple rule. But my emphasis is to develop this geometric tool, so only suggestions about how to use this approach to study behavioral issues are offered here; i.e., much more remains to be done with these techniques.

While this development requires a specified profile, the goal for other theoretical problems is to determine whether certain rules can exhibit certain specified properties. Can a profile be found, for instance, where DPV has election rankings that are directly opposite one another? If so, what are the characteristics of the profile? In Sect. 6, I create an approach to answer these kinds of questions.

While my conclusions extend to any number of candidates, I emphasize the threecandidate setting primarily so that I can use simple geometry to describe the ideas and expose hidden structures governing voting behavior. But the approaches developed here extend to any number of candidates in terms of the geometry of higher dimensional spaces. 


\section{Simple geometry}

It is obvious that a single profile can generate several different multiple rule election tallies and even rankings. After all, each choice of a positional rule that a voter can use contributes a different value to the final tally. What is surprising is the huge number of distinct tallies that can arise with even a modest number of voters. Indeed, there are so many outcomes that, to characterize what can happen, different approaches need to be created. The geometric technique introduced starting in Sect. 2.2 is first illustrated with AV (where the ideas are easier to follow).

\subsection{Computing outcomes}

The following eighteen voter profile is used to compare the outcomes allowed by various multiple rules. I start by finding all of its AV outcomes.

\begin{tabular}{c|c||c|c|c} 
Number & Ranking & $A$ & $B$ & $C$ \\
\hline 7 & $A \succ C \succ B$ & 7 & & $x$ \\
6 & $B \succ C \succ A$ & & 6 & $y$ \\
1 & $C \succ A \succ B$ & $z$ & & 1 \\
4 & $C \succ B \succ A$ & & $u$ & 4 \\
\hline & Total & $7+z$ & $6+u$ & $5+(x+y)$
\end{tabular}

Each row indicates how many voters with the specified preferences select the antiplurality rule. Thus the $x$ in the first row means that $x$ of the seven voters vote approval for two candidates. All possible AV tallies are obtained by using the different integer values of $0 \leq x \leq 7,0 \leq y \leq 6,0 \leq z \leq 1$, and $0 \leq u \leq 4$. By experimenting with $x, y, z, u$ choices, it follows that each of the thirteen ways to rank three candidates is an admissible AV outcome. To have an $A \sim B \sim C$ complete tie, for instance, let $z=y=0, u=1, x=2$. To compute the number of AV voting options, notice that there are eight $x$ values, seven $y$ values, two $z$ values and five $u$ values, so there are $8 \times 7 \times 2 \times 5=560$ different AV election scenarios. But as a $x, y$ choice leads to the same tally as a $x-1, y+1$ choice, the number of distinct tallies is smaller. The following definition is used to compute this value.

Definition 2 For three candidates, the Second Place Tallies (SPT) is a vector specifying the difference between each candidate's antiplurality (AP) and plurality (P) tallies. For four candidates, SPT is the vector of differences between each candidate's "vote-for-two" and plurality tallies, while the Third Place Tallies (TPT) is the vector of the differences between each candidate's AP (here, "vote-for-three") and "vote-for-two" tallies. For more candidates, higher place tallies are similarly defined.

With Eq. $4, P=(7,6,5)$ and $\mathrm{AP}=(8,10,18)$ so $\mathrm{SPT}=(8,10,18)-(7,6,5)=$ $(1,4,13)$. Candidate $A$ has the two possible tallies of 7 and 8 ; this value of two comes from adding one (where nobody with $A$ second ranked votes for her) to $A$ 's $S P T$ value of one. To find the number of distinct AV tallies add one to each SPT component and then multiply them together. Thus this example admits $(1+1) \times(4+1) \times(13+1)=$ 
140 different AV tallies. By comparing this value with the number of scenarios, it follows that, on average, each of the $140 \mathrm{AV}$ tallies can be attained in four different ways.

\subsubsection{RV}

The RV and AV rules differ in that RV replaces the "either-or" AV choices with a gradation. Because AV is a special RV case, it follows for Eq. 4 profile that any of the thirteen ways to rank the three candidates is an admissible RV election outcome. To reduce the number of different possible RV tallies (by a multiple of millions), assume that a voter always gives his top ranked candidate the largest allowable number of points. This assumption permits the natural scaling where each RV voter assigns 1 point to a top ranked candidate and any hundredths of points between 0 and 1 to a second ranked candidate; e.g., the $x$ in Eq. 4 can be any of the hundredths of points ranging between 0 and 7 . Thus this eighteen voter example admits over $700 \times 600 \times 100 \times 400=16,800,000,000$ different RV voting options. To compute the number of distinct RV tallies (with the normalization assumption), multiply each SPT component by 100 , add one, and multiply the values together to discover that this one profile admits $101 \times 401 \times 1301=52,691,801$ different RV election tallies. On average, then, each of the over 52 million different RV tallies can be attained in about 318 different ways.

\subsubsection{Truncated voting for strategic purposes}

As described earlier, by admitting truncated ballots, a multiple system is created. To illustrate how to analyze such a system, consider the earlier voting rule of $(5,2,0)$, where a truncated ballot means that the voter is using $(5,0,0)$. In the following table the $x, y, z$, and $u$ values indicate the number of voters who cast a truncated ballot.

\begin{tabular}{c|c||c|c|c} 
Number & Ranking & $A$ & $B$ & $C$ \\
\hline 7 & $A \succ C \succ B$ & $7 \times 5$ & & $2(7-x)$ \\
6 & $B \succ C \succ A$ & & $6 \times 5$ & $2(6-y)$ \\
1 & $C \succ A \succ B$ & $2(1-z)$ & & 5 \\
4 & $C \succ B \succ A$ & & $2(4-u)$ & $4 \times 5$ \\
\hline & Total & $37-2 z$ & $38-4 u$ & $51-2(x+y)$
\end{tabular}

"Even-odd" parity concerns make it impossible to have a $A \sim B$ or $B \sim C$ tie, which means that it is impossible to have five outcomes such as $A \sim B \succ C$ or $A \succ B \sim C$. But the eight remaining rankings are admissible. If truncated voting is strategically used to alter the sincere $C \succ B \succ A$ outcome, only the $B \succ C \succ A$ voters have an incentive to truncate their ballots. Even if all six did so, the outcome would remain the same. 


\subsubsection{CV}

The analysis differs for $\mathrm{CV}$. Using $x, y, z$, and $u$ integer values to designate the number of voters who select $(1,1,0)$ rather than $(2,0,0)$, all possible tallies become

\begin{tabular}{c|c||c|c|c} 
Number & Ranking & $A$ & $B$ & $C$ \\
\hline 7 & $A \succ C \succ B$ & $14-x$ & & $x$ \\
6 & $B \succ C \succ A$ & & $12-y$ & $y$ \\
1 & $C \succ A \succ B$ & $z$ & & $2-z$ \\
4 & $C \succ B \succ A$ & & $u$ & $8-u$ \\
\hline & Total & $14-x+z$ & $12-y+u$ & $10-(z+u)+(x+y)$
\end{tabular}

To prove that each of the thirteen ways to rank the candidates is an admissible CV election outcome, notice that a complete tie occurs with $x=2, y=z=u=0$. By varying the values from this choice, it follows that all other rankings can be created. Computing the number of $\mathrm{CV}$ distinct tallies involves combinatorics, but, for our purpose, notice that as $A$ 's vote can range from 7 to 15 , she can have 9 different tallies. Thus, while this profile generates a large number of CV tallies, it is less than $9 \times 11 \times 18=1782$.

Although this algebraic approach provides insights, it is not easy to use it to compare the AV, RV, and CV outcomes. This problem is addressed next.

\subsection{Convex hulls}

The different sets of election tallies could be compared by plotting each tally from each set in a three-dimensional space; a point's $(A, B, C)$ coordinates represent, respectively, the election tally for $A, B$, and $C$. But as Eq. 4 example demonstrates, with even a modest number of voters, the number of admissible election tallies quickly becomes unmanageable; e.g., it is unrealistic to determine and plot all 52 million different RV tallies that are associated with this one profile.

To handle this problem, the approach developed here is to describe the set of all election tallies. Rather than computing each admissible tally, the idea is to determine the convex hull of all possible election tallies that are defined by a specified profile $\mathbf{p}$. To conveniently compare diagrams, replace each candidate's election tally with the fraction of her total vote; e.g., replace the $(150,300,450)$ tally with $\left(\frac{150}{900}=\right.$ $\frac{1}{6}, \frac{300}{900}=\frac{1}{3}, \frac{450}{900}=\frac{1}{2}$ ) capturing, for instance, that $C$ received half of the vote. This choice replaces the complexity of comparing sets of election outcomes in a threedimensional space with a comparison of the structure of sets in the two dimensional dimensional simplex

$$
\mathcal{S}=\left\{(x, y, z) \in \mathbb{R}^{3} \mid x, y, z, \geq 0, x+y+z=1\right\},
$$

which has the shape of an equilateral triangle. In this manner, for instance, the set of 140 different AV tallies with the Eq. 4 example can be characterized by plotting just eight points! 
Definition 3 For a voting rule and a profile p, the voting rule's "tally hull" is the convex hull of all outcomes after each tally is normalized to represent the fraction of the total vote assigned to each candidate. It is denoted by $\mathcal{T} \mathcal{H}_{\text {voting rule }}(\mathbf{p})$.

As an illustration of this definition, it is reasonable to wonder whether a profile's plurality outcome, or Borda outcome, or $(6,5,0)$ outcome is an admissible CV outcome. Does RV admit election rankings that cannot occur with AV? Partial answers for these questions come from the following, which asserts, in part, that for any profile, the sincere election ranking for any positional method is, indeed, an admissible CV, or RV ranking:

Theorem 2 For any given profile $\mathbf{p}$ and $n \geq 3$ candidates, the following inequality holds:

$$
\mathcal{T} \mathcal{H}_{\text {positional rule }}(\mathbf{p}) \subset \mathcal{T} \mathcal{H}_{\mathrm{AV}}(\mathbf{p}) \subset \mathcal{T} \mathcal{H}_{\mathrm{CV}}(\mathbf{p})=\mathcal{T} \mathcal{H}_{\mathrm{DPV}}(\mathbf{p}) \subset \mathcal{T} \mathcal{H}_{\mathrm{RV}}(\mathbf{p})
$$

For any profile $\mathbf{p}$, then, each positional election ranking is an admissible AV ranking; each admissible AV ranking is an admissible CV ranking, and so forth. Moreover, in each comparison, profiles exist whereby the containment is strict. Thus, because Eq. 7 requires $\mathcal{T} \mathcal{H}_{\mathrm{AV}}(\mathbf{p}) \subset \mathcal{T} \mathcal{H}_{\mathrm{CV}}(\mathbf{p})$, there exist profiles where $\mathrm{CV}$ has election rankings that are not admitted by AV. A surprise (for me) is that $\mathcal{T} \mathcal{H}_{\mathrm{CV}}(\mathbf{p})=\mathcal{T} \mathcal{H}_{\mathrm{DPV}}(\mathbf{p})$; although DPV has infinitely more tallies than CV, the hulls agree!

Part of the proof of Theorem 2 is to show how to construct these hulls, how to compare the different hulls, and how to start with a desired tally hull and then determine whether it has a supporting profile.

\subsection{The procedure line}

A complete discussion requires reviewing the procedure line (Saari 1995). The construction involves the plurality and antiplurality tallies represented by $P=\left(P_{A}, P_{B}\right.$, $\left.P_{C}\right)$ and $\mathrm{AP}=\left(A_{A}, A_{B}, A_{C}\right)$ as well as the earlier defined SPT.

For a specified positional method $\left(w_{1}, w_{2}, 0\right)$, divide the components by $w_{1}$ to find the equivalent rule $\left(\frac{w_{1}}{w_{1}}, \frac{w_{2}}{w_{1}}, 0\right)$ with the $(1, s, 0)$ form for a specific value $s \in[0,1]$. Thus $s=0, \frac{1}{2}, 1$ represent, respectively, the plurality, Borda Count, and antiplurality vote. Another result (Saari 1995) asserts that a profile's normalized $\mathbf{w}_{s}=(1, s, 0)$ tally is given by

$$
(1-s) P+s \mathrm{AP}=P+s(\mathrm{AP}-P)=P+s S P T .
$$

Illustrating with Eq. 4 profile, the plurality and antiplurality outcomes are, respectively, $P=(7,6,5)$ and $\mathrm{AP}=(8,10,18)$, so the tally for any specific $s$ is

$$
(7,6,5)+s(8-7,10-6,18-5)=(7+s, 6+4 s, 5+13 s)
$$

e.g., the Borda tally $\left(s=\frac{1}{2}\right)$ is $\left(\frac{15}{2}, \frac{16}{2}, \frac{23}{2}\right)$, which, when multiplied by two, returns the standard $(2,1,0)$ Borda tally of $(15,16,23)$. 


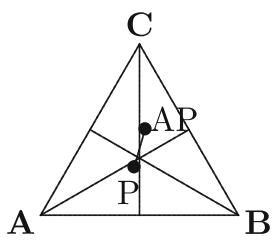

a

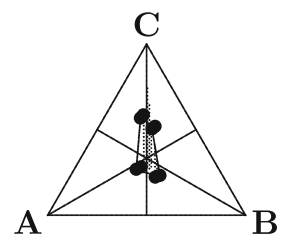

b

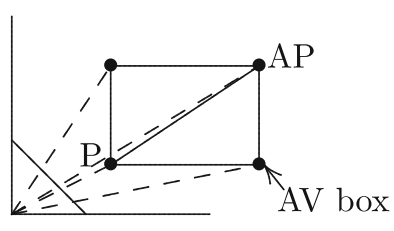

C

Fig. 1 Computing tally hulls. a Procedure line; b AV hull; c AV box and distortion effect

As Eq. 8 is a linear equation, all of a profile's positional outcomes are found by connecting the plurality and antiplurality tallies with a straight line. ${ }^{1}$ To make this construction easier to visualize, connect, instead, the normalized $\frac{P}{n}$ and $\frac{\mathrm{AP}}{2 n}$ tallies with a straight line. Each point on this procedure line is the normalized election tally for some positional outcome. However, the projection (the normalization) distorts the position of the $\mathbf{w}_{s}$ outcome from being $s$ th of the way to $\frac{2 s}{1+s}$ of the way from the plurality endpoint; ${ }^{2}$ e.g., the Borda $\left(s=\frac{1}{2}\right)$ outcome is midway between the plurality and antiplurality tallies in Eq. 8, but it is $\frac{2(1 / 2)}{1+(1 / 2)}=\frac{2}{3}$ of the way from the normalized plurality to antiplurality point on the procedure line. All of this is illustrated in Fig. 1a with Eq. 4 data. The distortion effect, as depicted in Fig. 1c, is a two-dimensional version of what happens in three-dimensions. (The slanted line $x+y=1$ represents Fig. 1a, $x+y+z=1$ plane.) The normalized image of any bullet is where the dashed line crosses the $x+y=1$ line. This projection pushes the midpoint of the line connecting $P$ with AP closer to AP on the projection; this is the described $\frac{2 s}{1+s}$ distortion effect.

The triangle's three reference lines connect an edge's midpoint to a vertex, so each represents a tied ranking for a particular pair; e.g., points on Fig. 1a vertical line have an $A \sim B$ tied outcome. Thus the center point represents the complete tie $A \sim B \sim C$, while the six open regions represent strict rankings. Figure 1a region with $P$ is closest to the $A$ vertex, next closest to $B$ and farthest from $C$, so the plurality ranking is $A \succ B \succ C$.

Figure 1a procedure line crosses seven regions, which means that by changing the choice of the positional method used to tally the ballots, this profile admits seven different election rankings; four of them are strict without ties. Notice how this line passes near the center point; this geometry requires the tallies for certain positional methods to come close to defining a complete tie. For examples how to use this procedure line and its generalization of a procedure hull (for more candidates), see Nurmi (2002), Saari (2001a,b), Tabarrok (2001), and Tabarrok and Spector (1999).

\footnotetext{
1 With four or more candidates, replace the procedure line with the procedure hull; its vertices are given by the normalized vote-for-one, vote-for-two, vote-for-three, ...election outcomes. See Saari (1992, 2001a).

2 A normalized tally divides a candidate's tally by the total vote. Similarly, the normalized value of $\mathbf{w}_{s}=$ $(1, s, 0)$ is $\left(\frac{1}{1+s}, \frac{s}{1+s}, 0\right)$, so the distance from the plurality endpoint is a multiple of $\frac{s}{1+s}$. As $s=1$ is the AP endpoint with value $\left(\frac{1}{2}, \frac{1}{2}, 0\right)$, the distance of $\mathbf{w}_{s}$ on the procedure line is $2 \frac{s}{1+s}$.
} 


\subsection{Computing the AV hull}

The AV hull is completely determined by the $P$ and AP values. To appreciate what is involved, notice from Eq. 4 that the AV tallies for the candidates range from the plurality outcome of $P=(7,6,5)$, where nobody votes for his second ranked candidate, to the antiplurality outcome of $\operatorname{AP}=(8,10,18)$, where everyone votes for two candidates. Thus each candidate's admissible tallies vary in increments of 1 ranging from her plurality to her antiplurality tally. The combinations of each candidate's two extreme tallies define eight extreme tallies that define the vertices of a box; call it the $A V$ box. The eight vertices for this example are

$$
\begin{array}{rlrr}
(7,6,5), & (7,6,18), & (7,10,5), & (8,6,5), \\
(8,10,18), & (8,10,5), & (8,6,18), & (7,10,18) .
\end{array}
$$

The normalized version of these tallies, where the sum of the components equals unity, are plotted in Fig. 1b. These plotted points are

$$
\begin{array}{llll}
\left(\frac{7}{18}, \frac{6}{18}, \frac{5}{18}\right), & \left(\frac{7}{31}, \frac{6}{31}, \frac{18}{31}\right), & \left(\frac{7}{22}, \frac{10}{22}, \frac{5}{22}\right), & \left(\frac{8}{19}, \frac{6}{19}, \frac{5}{19}\right), \\
\left(\frac{8}{36}, \frac{10}{36}, \frac{18}{36}\right), & \left(\frac{8}{23}, \frac{10}{23}, \frac{5}{23}\right), & \left(\frac{8}{32}, \frac{6}{32}, \frac{18}{32}\right), & \left(\frac{7}{35}, \frac{10}{35}, \frac{18}{35}\right) .
\end{array}
$$

The AV convex hull is created by connecting the eight points with straight lines. As the tallying process of Eq. 4 requires the 140 different AV tallies to differ from a neighbor by one vote, the tallies are uniformly distributed in the AV box. Thus the normalized tallies are "essentially" uniformly distributed (but not quite because of the distortion associated with the normalization) within the AV hull. This association between the AV box and the AV hull is depicted in Fig. 1c.

To construct the AV hull, first compute the plurality and antiplurality tallies for a given profile. Next find the eight extreme points defined by the $P=\left(P_{A}, P_{B}, P_{C}\right)$ and $\mathrm{AP}=\left(A_{A}, A_{B}, A_{C}\right)$ values. Plot the normalized values of these eight points; the associated convex hull is the AV hull.

For a sample of the kinds of results that can be obtained from the geometry, notice that the AV hull always includes the plurality and antiplurality tallies. Thus the procedure line always is a subset of $\mathcal{T} \mathcal{H}_{\mathrm{AV}}$, which means that anything that can occur with any positional voting rule can also occur with AV. This statement proves the first part of Eq. 7 string of inclusions

$$
\mathcal{T} \mathcal{H}_{\text {positional rule }}(\mathbf{p}) \subset \mathcal{T} \mathcal{H}_{\mathrm{AV}}(\mathbf{p})
$$

The assertion that there are AV outcomes that cannot occur with any positional method is illustrated by Fig. 1b: each of the thirteen possible ways to rank the candidates is an admissible AV outcome, but only seven of these rankings can be positional outcomes. With this profile, then, six AV election rankings cannot occur with any positional rule. 


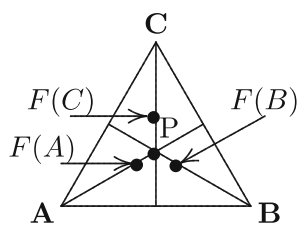

a

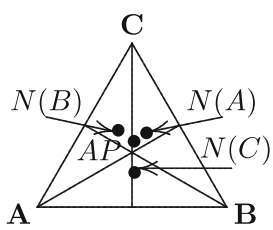

b

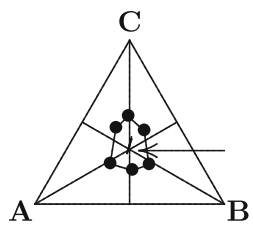

c

Fig. 2 Explaining AV hulls. a $P(X)$ points; b $A(X)$ points; c AV hull

\section{Using the hulls}

A convenient way to examine AV behavioral consequences is to first label the vertices of the AV hull. By doing so, a simple rule emerges that completely determines when some candidate could beat others in an AV election. Finally, the structure of the AV hull is completely described in terms of the profile decomposition. For instance, the $\mathrm{AV}$ hull of Fig. $1 \mathrm{~b}$ is quite narrow. Is this true in general? What parts of a profile determine the shape and size of the hull?

\subsection{Behavioral coordinates}

The eight points defined by the plurality and antiplurality tallies of $P=(17,16,15)$ and $\mathrm{AP}=(28,30,38)$ are listed in Eq. 11 with titles. Here $F(X)$ changes the plurality tally in that everyone with candidate $X$ second-ranked votes for her, but no other candidate receives second place votes. $N(X)$ changes the antiplurality tally in that only candidate $X$ does not receive any second place votes; each of the other two candidates receives all possible second place votes.

$$
\begin{aligned}
& P=(17,16,15), \quad F(C)=(17,16,38), \quad F(B)=(17,30,15), \\
& F(A)=(28,16,15), \quad \mathrm{AP}=(28,30,38), \quad N(C)=(28,30,15), \\
& N(B)=(28,16,38), \quad N(A)=(17,30,38) \text {. }
\end{aligned}
$$

These eight points define the vertices of a rectangular AV box. The normalized $P$ and $F(X)$ values are plotted in Fig. 2a, the normalized AP and $N(X)$ values are in Fig. 2b, the AV hull is in Fig. 2c. The plurality and antiplurality points are the endpoints of the short procedure line hiding in the interior of the hull near the center point.

Each $F(X)$ point is on the line from $P$ toward the $X$ vertex. $^{3}$ As such, if each $S P T$ component is non-zero (meaning each candidate is second ranked by at least one voter), then the plurality outcome is an interior point of the convex hull defined by the $F(X)$ points. Similar statements hold for the $N(X)$ points except that $N(X)$ is located on the line through AP away from the $X$ vertex.

Theorem 3 If each SPT component is non-zero, then $P$ is in the interior of the hull defined by the normalized $F(X)$ points, AP is in the interior of the hull defined by

\footnotetext{
3 A proof of this assertion is immediate; as only $X$ receives second place votes, this edge on the rectangular box is parallel to the $X$-axis. The projection argument now follows.
} 
the normalized $N(X)$ points, and the procedure line is strictly in the interior of the $A V$ hull. ${ }^{4}$ Consequently, with these assumptions, the procedure line is strictly in the interior of the CV, DTP, and RV hulls.

The last part of this theorem follows from Theorem 2.

Notice the ordering of the $F(X)$ and $N(X)$ points in Fig. 2c AV hull. Starting with $F(A)$ and moving in a counter-clockwise direction, the points are $F(A), N(C), F(B)$, $N(A), F(C), N(B), F(A)$. As I will explain in Sect. 3.3, this ordering always occurs. Also notice that behavior favoring some candidate, say $A$, is represented by the portion of a profile in the triangle with vertices $F(A), N(B), N(C)$.

Strategic behavior and other choices. Earlier I asked for an interpretation of an AV tally of $(18,29,16)$ for the Eq. 1 profile. This profile's AV hull is Fig. 2c, where the particular tally is near the $F(B)$ vertex. Thus, to have this AV outcome, it follows from the geometry that $B$ must be the only candidate to receive a large number of second ranked votes. On the other hand, because the AV tally of $(22,23,26)$ is near the center of the hull, many different symmetrical combinations support this AV outcome.

In other words, a way to approach a behavioral analysis is to recognize that the $F(X)$ and $N(X)$ terms identify which voters vote for one or two candidates. In Sect. 2, we discovered, by comparing the number of scenarios with the number of different tallies, that the points in this AV hull can be obtained in several different ways; namely, there are different scenarios that describe why the same election outcome can arise.

To indicate how to use these coordinates, suppose an AV outcome is at Fig. 2c arrow tip in the $B \succ C \succ A$ ranking region; the procedure line is not in this region, so this AV outcome differs from all possible positional rankings. The geometry indicates what combinations of voter behaviors support this outcome. One Fig. 2a choice involves a combination of $F(B)$ and $F(C)$ outcomes meaning that $A$ is not picking up many second place votes. A different scenario, coming from Fig. $2 \mathrm{~b}$, is where the profile has a strong collection of $N(A)$ voters, who do not give $A$ second place votes, but also $N(C)$ voters, where, with this partition set, $A$ receives second place votes but $C$ does not. Namely, labeling the vertices makes it easier to identify the different ways in which voters can be divided - the different behavioral scenarios-to explain each tally.

The geometry also shows that with a small number of strategic voters, opportunities for strategic action arise when a sincere election outcome is near an indifference line. (An immediate corollary is that a longer indifference line in an AV hull provides more strategic opportunities.) In Fig. 2c, if the sincere outcome is in the $B \succ C \succ A$ region near the $B \sim C$ indifference line, then opportunities exist to strategically elect $C$ rather than $B$. Voters who would benefit prefer $C \succ B$; i.e., voters with $A \succ C \succ B, C \succ A \succ B$ and $C \succ B \succ A$ preferences.

According to Fig. 2c, if voters vote strategically by voting for one or for two candidates (i.e., without misrepresenting preferences), the successful strategic actions are those that move the outcome toward the $N(B), F(C)$, and $N(A)$ points. Thus, only

\footnotetext{
4 A similar result for any number of candidates asserts that the procedure hull is strictly in the interior of the AV hull. The added conditions are the obvious ones; e.g., for four candidates, each component of the third place tallies must be non-zero.
} 
those $A \succ C \succ B$ voters who sincerely voted for one candidate have a strategy: vote for two to move the outcome toward the $F(C)$ point. For the other two types, only the voters who would sincerely vote for two have a strategy: vote for one to move the outcome toward the $N(B)$ and $N(A)$ points.

\subsection{Who can beat whom?}

Next, I use this geometry to create a simple way to determine all admissible AV election rankings. For intuition, it is reasonable to expect that the AV bottom ranked candidate is someone who is not highly ranked by most voters. The next definition pushes this notion to an extreme by excluding only those candidates who are bottom ranked by too many voters.

Definition 4 Candidate $X$ is "in the mix" with candidate $Y$ if the number of voters who do not have $X$ bottom ranked is more than the number of voters who have $Y$ top ranked.

Suppose $A$ and $B$ receive, respectively, 45 and $40 \%$ of the first-place rankings, while $C$ is bottom ranked by $51 \%$ of the voters. As $49 \%$ of the voters do not have $C$ bottom ranked, she is in the mix with $A$ and $B$. As $A$ is not bottom ranked by at least $45 \%$ of the voters and as $C$ is top ranked by $15 \%, A$ is in the mix with everyone; as $B$ is not bottom ranked by at least $51 \%$ of the voters (who have $C$ bottom ranked), she too is in the mix.

The next theorem provides a quick way to find all possible admissible AV rankings.

Theorem 4 For a three-candidate profile, if $X$ is in the mix with $Y$, there exists sincere $A V$ election outcomes where $X$ beats $Y$. If each of the candidates is in the mix with any other candidate, then all possible ways to rank the candidates is an admissible $A V$ election outcome. But if $X$ is not in the mix with $Y$, then no AV outcome can rank $X$ above $Y$.

The proof follows from the construction of the AV hull. If $X$ is in the mix with $Y$, then at least one vertex for the convex hull has a larger $X$ than a $Y$ component, so there is at least one ranking where $X \succ Y$. Conversely, if $X$ is not in the mix with $Y$, then, because all of the AV hull's vertices have the $X$ component bounded by the $Y$ component, the convex hull cannot enter a region where $X$ beats $Y$. A slightly modified condition holds for any number of candidates.

The example prior to Theorem 4 has everyone is in the mix, so any of the 13 transitive ways to rank three candidates is an admissible AV outcome. It follows from Eq. 11 that for three candidates the $F(X)$ tallies completely determine with whom $X$ is in the mix.

\subsection{Using the profile decomposition}

Figure 2c AV hull is broader than the one in Fig. 1b even though the P and AP tallies (from Eqs. 4 to 11) differ only by adding 10 votes to each candidate. It is reasonable 


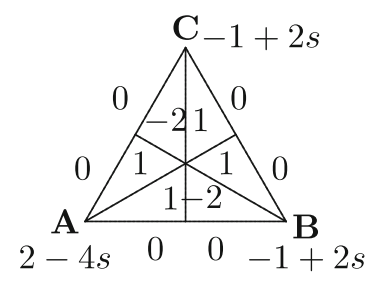

a

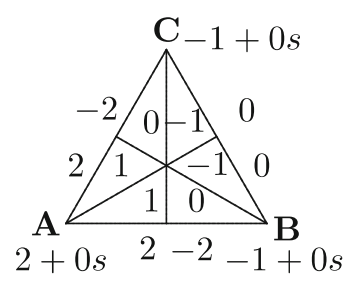

b

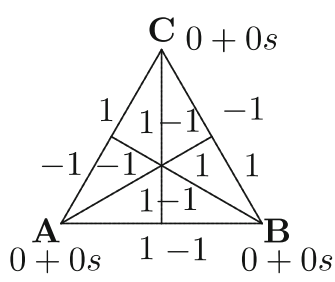

c

Fig. 3 A profile basis. a $A$-Reversal; $\mathbf{R}_{A} ; \mathbf{b} A$-Basic; $\mathbf{B}_{A}$; c Condorcet; $\mathbf{C}^{3}$

to wonder what causes differences in the shape and size of AV hulls. Answers come from the profile decomposition, where a profile is divided into the components that have different effects on election outcomes. Three of the six basis directions for this decomposition are given in Fig. 3.

Each triangle ranking region lists the number of voters with that particular ranking. In Fig. 3a, then, each of four rankings has a single voter while there are -2 voters with the $C \succ A \succ B$ and $B \succ A \succ C$ preferences. The negative values, which require the sum of the components in a profile component to equal zero, are needed to ensure the orthogonality among the four different profile directions; this orthogonality is what separates the portions of a profile into the parts that cause different kinds of voting effects. For an intuitive, practical interpretation, treat the negative values as describing how many voters changed preferences from the indicated ranking to one with a positive component. So, treat Fig. $3 \mathrm{a}$ as describing that two voters changed from each of the $C \succ A \succ B$ and $B \succ A \succ C$ preferences to adopt one of the four other rankings.

Three of the six-dimensions of profile space are listed above. A fourth is the Kernel, $\mathbf{K}$, which assigns one voter to each of the six rankings. A fifth direction is the B-Basic, $\mathbf{B}_{B}$, direction given by modifying Fig. 3b; i.e., assign a single voter to each ranking where $B$ is top-ranked and -1 voters for each ranking where $B$ is bottom ranked. (As $\mathbf{B}_{A}+\mathbf{B}_{B}+\mathbf{B}_{C}=\mathbf{0}$, any two Basic directions suffice. This expression allows components with negative coefficients to be converted into one with non-negative coefficients; e.g., $3 \mathbf{B}_{A}-2 \mathbf{B}_{B}=3 \mathbf{B}_{A}+2\left(\mathbf{B}_{A}+\mathbf{B}_{C}\right)$.) The final profile direction is $B$-Reversal, $\mathbf{R}_{B}$, which mimics the construction of $\mathbf{R}_{A}$ by assigning a single voter for each ranking where $B$ is top or bottom ranked and -2 voters for each ranking where $B$ is middle ranked. Again, $\mathbf{R}_{A}+\mathbf{R}_{B}+\mathbf{R}_{C}=\mathbf{0}$, so any two reversal directions suffice.

All possible profiles can be expressed as a linear combination of these profile directions (Saari 1999). For instance, the Condorcet triplet

$$
\{B \succ A \succ C, A \succ C \succ B, C \succ B \succ A\}=\frac{1}{2}\left[\mathbf{K}-\mathbf{C}^{3}\right] .
$$

The $\frac{1}{2} \mathbf{K}$ term states that there are three voters; the Condorcet term rearranges preferences; e.g., a voter with an $A \succ B \succ C$ ranking in $\mathbf{K}$ is moved to another ranking. A matrix expression to convert a profile into its component parts is in Saari (1999, 2008). 


\subsubsection{Interpreting profile directions}

The importance of these directions is captured by the expressions posted by the vertices and under each leg of the triangle. To compute the pairwise tallies for a pair, count the number of points on each side of the appropriate indifference line. In Fig. 3a, for instance, the $A-B$ tally is the sum of points on each side of the vertical line: the $0-0$ tally means that each of these two candidates receives zero votes. The values by the vertices show the $\mathbf{w}_{s}$ outcomes; they are obtained by adding the numbers in the two regions that share the candidate's vertex and adding $s$ times the SPT values, which is the sum of numbers in the two regions adjacent to the top-ranked regions.

These values show that all positional and majority vote outcomes completely agree on the Basic components of a profile. Thus, all differences in positional methods are due to Reversal components (which do not effect pairwise rankings), and all differences in pairwise rankings are caused by the Condorcet component (which has no effect on positional rankings). For more information, see Saari $(1999,2008)$.

According to the above, adding multiples of $\mathbf{C}^{3}$ to a profile does not affect the positional tallies. Illustrating with Eq. 1 profile, adding all possible $-x \mathbf{C}^{3}$ terms that do not leave a negative component creates eleven different, but related, profiles (for $0 \leq x \leq 10$ ) that have precisely the same tallies for each positional rule.

\begin{tabular}{cc|cc} 
Number & Ranking & Number & Ranking \\
\hline $10-x$ & $A \succ B \succ C$ & $4+x$ & $C \succ B \succ A$ \\
$7+x$ & $A \succ C \succ B$ & $16-x$ & $B \succ C \succ A$ \\
$11-x$ & $C \succ A \succ B$ & $0+x$ & $B \succ A \succ C$
\end{tabular}

By having the same positional tallies, all 11 of these profiles share Fig. 2c AV hull. Thus, the same AV hull holds for any positive multiple of these profiles.

\subsubsection{Profile components that affect the AV box}

To determine which parts of a profile influence the shape of the AV box, compute $F(X)$ and $N(X)$ for each profile component. As asserted previously, the $F(X)$ and $N(X)$ are not affected by a profile's Condorcet component, both vertices have precisely the same value on a profile's Basic component, and both have precisely opposite effects on a profile's Reversal and Kernel elements. Thus the four diagonal vertices of the AV box are $\{P, \mathrm{AP}\}$, and $\{F(X), N(X)\}$ for $X=A, B, C$. It also follows that the midpoint for each of these diagonals is the Borda score; namely the Borda outcome is symmetrically located at the AV box's center. In $\mathcal{T} H_{\mathrm{AV}}(\mathbf{p})$, the line connecting any $F(X)$ with $N(X)$ crosses the procedure line precisely at the normalized Borda tally.

Theorem 5 a. The Borda tally is at the midpoint of the four diagonals of the AV box.

b. The $F(X), N(X)$, plurality, and antiplurality tallies of $\mathbf{C}^{3}$ are zero.

c. The $F(X), N(X)$, plurality, antiplurality, and any $\mathbf{w}_{S}$ tally of any basic profile component, $a_{B} \mathbf{B}_{A}+b_{B} \mathbf{B}_{B}$ for any scalars $a_{B}, b_{B}$, completely agree.

d. The $\mathbf{w}_{s}$ outcome of $\mathbf{K}$ assigns $2+2$ s points to each candidate, leading to a complete tie. The $F(X)$ outcome of $\mathbf{K}$ differs from the Borda tally of $(3,3,3)$ by 


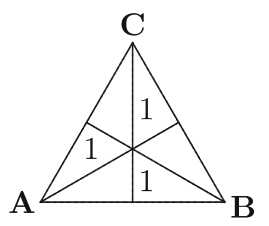

$\mathbf{a}$

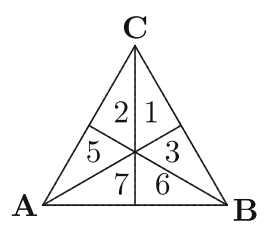

b

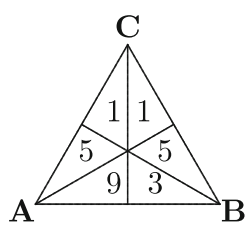

c

Fig. 4 Examples. a $\frac{1}{2}\left[\mathbf{K}-\mathbf{C}^{3}\right] ; \mathbf{b} 4 \mathbf{K}+\left[3 \mathbf{B}_{A}+2 \mathbf{B}_{B}\right] ; \mathbf{c}$ Plus $\mathbf{R}_{A}+\mathbf{C}^{3}$

adding 1 point to $X$ and -1 points to each of the other two candidates. The $N(X)$ outcome differs from the Borda tally of $(3,3,3)$ by adding -1 points to $X$ and 1 points to each of the other two candidates. Thus the $F(X)$ and $N(X)$ values are symmetrically located relative to the $(3,3,3)$ Borda $\left(s=\frac{1}{2}\right)$ tally of $\mathbf{K}$.

e. The $F(X)$ tally for $\mathbf{R}_{X}$ assigns -2 points to $X$ and -1 to each of the two other candidates; the $N(X)$ tally is the reverse by assigning 2 points to $X$ and 1 to each of the two other candidates. The $F(X)$ tally of $\mathbf{R}_{Y}, X \neq Y$, assigns 1 point to $X, 2$ points to $Y$, and -1 points to the remaining candidate. The $N(X)$ value is the reverse by assigning -1 points to $X,-2$ points to $Y$, and 1 point to the remaining candidate. Thus the $F(X)$ and $N(X)$ vertices on a Reversal component are symmetric relative to the Borda tally.

For a proof, just compute the $F(X)$ and $N(X)$ values for the different Fig. 3 directions. Because the $F(X)$ and $N(X)$ terms have opposite values on Reversal terms, it follows for any profile where the Reversal terms dominate the Basic terms that the AV hull includes all 13 rankings. Conversely, if the AV hull does not include all thirteen rankings, then the Basic terms dominate.

Stated in other words, the many different AV tallies are strictly due to the Reversal and Kernel directions. The Kernel direction changes all $F(X)$ and $N(X)$ pairs in the same way (Theorem 5d) creating a cube centered about the Borda tally. Therefore, the crucial symmetries that characterize different AV outcomes are the Reversal components; they distort the cube into a rectangular box centered about the Borda tally. All of this is illustrated with the three profiles of Fig. 4.

With Fig. 4a Condorcet triplet $\frac{1}{2} \mathbf{K}-\frac{1}{2} \mathbf{C}^{3}$ of Eq. 13, the Condorcet term does not affect the $\mathbf{w}_{s}, F(X)$, or $N(X)$ terms (Theorem $5 \mathrm{~b}$ ), so all of the AV different tallies are due to the kernel term. On $\frac{1}{2} \mathbf{K}, P=(1,1,1), \mathrm{AP}=(2,2,2)$, and the Borda tally is the midpoint of $\left(\frac{3}{2}, \frac{3}{2}, \frac{3}{2}\right)$ (Theorem $5 \mathrm{~d}$ ). According to Theorem 5e, we have that $F(A)=\frac{1}{2}(1,-1,-1)+\left(\frac{3}{2}, \frac{3}{2}, \frac{3}{2}\right)=(2,1,1), N(A)=\frac{1}{2}(-1,1,1)+\left(\frac{3}{2}, \frac{3}{2}, \frac{3}{2}\right)=$ $(1,2,2)$ with a similar expression for the other terms. Thus the AV cube is centered about the Borda tally.

To analyze Fig. 4b example of $\mathbf{p}_{1}=\left[3 \mathbf{B}_{A}+2 \mathbf{B}_{B}\right]+4 \mathbf{K}$ in Fig. 4b, all $F(X), N(X)$, and $\mathbf{w}_{S}$ tallies agree on the bracketed terms (Theorem $5 \mathrm{c}$ ), so all $\mathrm{AV}$ differences reflect the $4 \mathbf{K}$ term. This requires the $\mathrm{AV}$ box to be a cube centered about the Borda $\left(s=\frac{1}{2}\right)$ tally of $(16,13,7)$. Thus (Theorem $\left.5 \mathrm{~d}\right)$, we have that $F(A)=4(1,-1,-1)+$ $(16,13,7)=(20,9,3)$ with similar terms for the other AV cube vertices. 
Figure $4 \mathrm{c}$ profile differs from Fig. $4 \mathrm{~b}$ profile by a $\mathbf{R}_{A}+\mathbf{C}^{3}$ term. The sum of coordinates in $\mathbf{R}_{A}+\mathbf{C}^{3}$ is zero, so Fig. $4 \mathrm{~b}$,c profiles have the same number of voters; some just shifted preferences. Again (Theorem $5 b$ ), the $\mathbf{C}^{3}$ term plays no role in the AV outcomes. Because the Borda Count is not affected by Reversal or Condorcet terms, the Borda tally remains $(16,13,7)$. The $\mathbf{R}_{A}$ term, however, changes the shape of the AV box from cubic to rectangular.

To see the shape change, starting with Fig. $4 \mathrm{~b}$ vertex, which includes the $4 \mathbf{K}$ effects, the new Fig. $4 \mathrm{c}$ vertex is $F(A)=(20,9,3)+(-2,-1,-1)=(18,8,2)$ while $N(A)=(12,17,11)+(2,1,1)=(14,18,12)$. This change contracts $A$ 's range of AV tallies but expands the possible $B$ and $C$ AV tallies. For the next diagonal, $F(B)=$ $(12,17,3)+(2,1,-1)=(14,18,2)$ while $N(B)=(20,9,11)+(-2,-1,1)=$ $(18,8,12)$ providing slightly wider choices for $B$ and $C$ tallies with fewer $A$ choices. Finally, we have $F(C)=(12,9,11)+(2,-1,1)=(14,8,12)$ while $N(C)=$ $(20,17,3)+(-2,1,-1)=(18,18,2)$ giving slightly more flexibility for $B$ 's AV tallies with tighter regions for the other two candidates.

\section{Computing the CV hull}

The CV hull is slightly more complicated to compute, but certain relationships are immediate. For instance, because the three choices offered by $\mathrm{CV}$ allow a voter to select the two AV options of $(1,0,0)$ or $(1,1,0)$, it follows immediately that $\mathcal{T} \mathcal{H}_{\mathrm{AV}} \subset$ $\mathcal{T} \mathcal{H}_{\mathrm{CV}}$.

The construction of the CV hull is based on the number of voters with the $j$ th preference, $x_{j}$, that elect to use $(1,1,0)$, rather than $(2,0,0)$, to tally ballots. Label the preferences as:

\begin{tabular}{cc|cc} 
Label & Ranking & Label & Ranking \\
\hline 1 & $A \succ B \succ C$ & 4 & $C \succ B \succ A$ \\
2 & $A \succ C \succ B$ & 5 & $B \succ C \succ A$ \\
3 & $C \succ A \succ B$ & 6 & $B \succ A \succ C$
\end{tabular}

With the profile $\mathbf{p}=\left(p_{1}, \ldots, p_{6}\right)$, we have that $0 \leq x_{j} \leq p_{j}$ with the plurality and antiplurality tallies of

$$
\begin{array}{ccc}
P_{A}=p_{1}+p_{2}, & P_{B}=p_{5}+p_{6}, & P_{C}=p_{3}+p_{4}, \\
A_{A}=P_{A}+\left(p_{3}+p_{6}\right), & A_{B}=P_{B}+\left(p_{1}+p_{4}\right), & A_{C}=P_{C}+\left(p_{2}+p_{5}\right) .
\end{array}
$$

The algebraic representation for all CV tallies for any specified profile is in Eq. 16. The first row, for instance, identifies the choices made by those voters who have $A$ top-ranked. If all of these voters select $(2,0,0)$ to tally the ballots, then $A$ 's tally from them is $2 P_{A}$. The $x_{1}$ means that $x_{1}$ of the $p_{1}$ voters (voters with the first kind of preference ranking, or, from Eq. $14, A \succ B \succ C$ ) decide to vote by using $(1,1,0)$. With these $x_{1}$ voters, $A$ loses $x_{1}$ points from her tally, while $B$ gains this amount. In general, the tallies are 


\begin{tabular}{c|c|c|c} 
& $A$ & $B$ & $C$ \\
\hline$A$ & $2 P_{A}-\left(x_{1}+x_{2}\right)$ & $x_{1}$ & $x_{2}$ \\
$B$ & $x_{6}$ & $2 P_{B}-\left(x_{5}+x_{6}\right)$ & $x_{5}$ \\
$C$ & $x_{3}$ & $x_{4}$ & $2 P_{C}-\left(x_{3}+x_{4}\right)$ \\
\hline Total & $2 P_{A}-\left(x_{1}+x_{2}\right)+\left(x_{3}+x_{6}\right)$ & $2 P_{B}-\left(x_{5}+x_{6}\right)+\left(x_{1}+x_{4}\right)$ & $2 P_{C}-\left(x_{3}+x_{4}\right)+\left(x_{2}+x_{5}\right)$
\end{tabular}

\subsection{Finding the CV polyhedron and hull}

As with the AV hull and the procedure line, a way to determine the CV hull is to first compute the $C V$ polyhedron as determined by its extreme tallies. Candidate $A$ 's smallest tally occurs when $x_{3}=x_{6}=0$ and $x_{1}=p_{1}, x_{2}=p_{2}$, yielding a $2 P_{A}-\left(p_{1}+\right.$ $\left.p_{2}\right)=P_{A}$ tally. As this value is independent of the $x_{4}, x_{5}$ values, the tallies are of the $\left(P_{A}, 2 P_{B}+p_{1}+\left(x_{4}-x_{5}\right), 2 P_{C}+p_{2}-\left(x_{4}-x_{5}\right)\right)$ form. Because of the common $\left(x_{4}-x_{5}\right)$ variable, these tallies define a line with the endpoints $\left(P_{A}, P_{B}+A_{B}, P_{C}+\right.$ $\left.\left(n-A_{B}\right)\right)$ and $\left(P_{A}, P_{B}+\left(n-A_{C}\right), P_{C}+A_{C}\right)$ where $n$ is the number of voters. The $P_{B}+\left(n-A_{C}\right)$ value occurs when $x_{3}=x_{6}=x_{4}=0, x_{1}=p_{1}, x_{2}=p_{2}, x_{5}=p_{5}$, which leads to the $B$ tally of $2 P_{B}-p_{5}+p_{1}=P_{B}+p_{6}+p_{1}=P_{B}+\left(n-A_{C}\right)$.

Similarly, $A$ 's largest tally occurs with $x_{1}=x_{2}=0, x_{3}=p_{3}, x_{6}=p_{6}$ with the value $2 P_{A}+\left(p_{3}+p_{6}\right)=P_{A}+A_{A}$. Again, the tallies accompanying this extreme value define a line defined by the variable $\left(x_{4}-x_{5}\right)$; the endpoints of this line accompanying this extreme $A$ tally are $\left(P_{A}+A_{A}, P_{B}, P_{C}+\left(n-A_{A}\right)\right)$ and $\left(P_{A}+A_{A}, P_{B}+(n-\right.$ $\left.\left.A_{A}\right), P_{C}\right)$.

Associated with each candidate are four extreme points; with combinations, they define 12 extreme points. The $P_{X}$ and $P_{Y}+A_{Y}$ values, however, are in all extreme tallies (where a maximum tally for one candidate is accompanied by a minimum one for another candidate), so each extreme point appears twice. Thus, both the CV polyhedron and $\mathcal{T} \mathcal{H}_{\mathrm{CV}}$ are defined by six extreme points. For purposes of labeling a graph (to facilitate an analysis of behavior possibilities), let $L(X)=P_{X}$ and $H(X)=P_{X}+A_{X}$ represent, respectively, candidate $X$ 's lowest possible and highest possible tally. Let $M(X)$ be the value of $X$ associated with an extreme point when the other two coordinates are low and high; notice, the definition of $M(X)=P_{X}+\left(n-A_{Y}\right)$ depends on which candidate $(Y)$ has the highest possible tally. The six vertices that determine the $\mathrm{CV}$ polyhedron are $(M(A), L(B), H(C)),(L(A), M(B), H(C)),(L(A), H(B)$, $M(C)),(M(A), H(B), L(C)),(H(A), M(B), L(C))$, and $(H(A), L(B), M(C))$. These points are listed in a clockwise ordering in Fig. 5 starting from the indicated vertex.

The sum of the tallies for each $\mathrm{CV}$ extreme point is $2 n$ where $P_{A}+P_{B}+P_{C}=n$ is the number of voters. This $2 n$ value arises because each of the $n$ voters has two points to distribute, ${ }^{5}$ e.g., for the $(L(A), H(B), M(C))$ extreme point,

$$
P_{A}+\left(P_{B}+A_{B}\right)+\left(P_{C}+\left(n-A_{B}\right)\right)=P_{A}+P_{B}+P_{C}+n=2 n .
$$

Thus the associated normalized tallies have the $\left(\frac{P_{A}}{2 n}, \frac{P_{B}+A_{B}}{2 n}, \frac{P_{C}+\left(n-A_{B}\right)}{2 n}\right)$ form.

\footnotetext{
5 At this stage, I am ignoring the voter's option of using $(1,0,0)$.
} 
Fig. 5 CV hull. a Equation 4 profile; b More typical profile

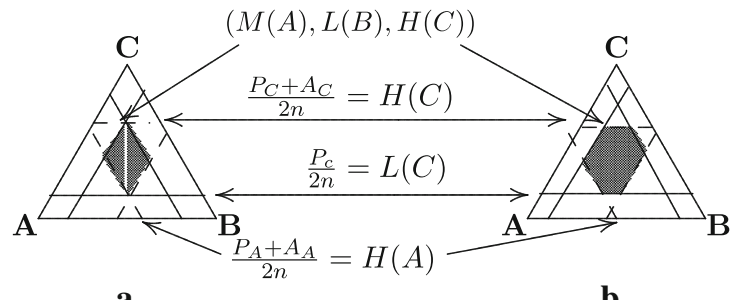

a b

To find the CV hull, notice that each normalized extreme point has a $L(X)=\frac{P_{X}}{2 n}$ tally. So in the simplex, draw the three lines given by these tallies; they are represented by the three solid lines in Fig. 5a, b. The horizontal solid line, for instance, represents all normalized tallies where $C$ receives $\frac{P_{C}}{2 n}$ points - this is half of $C$ 's normalized plurality tally of $\frac{P_{C}}{n}$ points. All normalized extreme points include a $H(X)=\frac{P_{X}+A_{X}}{2 n}$ tally, so plot these three lines; they are represented by the dashed lines in Fig. 5a. Because $\frac{P_{X}+A_{X}}{2 n} \geq \frac{A_{X}}{2 n}$, this normalized value is larger than what the candidate would receive with the normalized antiplurality vote.

The three dashed and solid lines intersect in six points (the two horizontal lines in Fig. 5a do not meet the crossing diagonal lines, so this is where two other points are located), these are the six extreme points that define the $\mathcal{T} \mathcal{H}_{\mathrm{CV}}$ hull. The shaded region in Fig. 5a is the hull for Eq. 4 profile; Fig. 5b depicts what happens with a more typical profile.

\subsection{Geometry}

As described next, the CV polyhedron is symmetrically centered about the $\left(\frac{3}{2}, \frac{1}{2}, 0\right)$ tally - the average of the $(2,0,0)$ and $(1,1,0)$ choices. (Rule $\left(\frac{3}{2}, \frac{1}{2}, 0\right)$ is the appropriate multiple of the $\mathbf{w}_{\frac{1}{3}}=\left(1, \frac{1}{3}, 0\right)$ where the sum of the $n$ voters' tallies equals $2 n$ so the outcome is in the polyhedron.) The opposing diagonals are of the $(M(A), L(B), H(C)$ and $(M(A), H(B), L(C))$ forms where two alternatives alternate in being high and low. The midpoint of any diagonal connecting diagonally opposing vertices is the $\frac{1}{2}(3,1,0)$ tally. Thus, to find the polyhedron, just compute the differences from the central tally.

With $n$ voters, the sum of $\mathbf{w}_{s}$ tallies is $n(1+s)$. As $2 n$ is the sum of tallies of any point in the $\mathrm{CV}$ polyhedron, no $\mathbf{w}_{s}$ tally, $s<1$, is in this part of the $\mathrm{CV}$ polyhedron. But with the $\mathrm{CV}$ hull, where tallies are projected to fractions of votes, the procedure line lies in the interior of $\mathcal{T} \mathcal{H}_{\mathrm{CV}}(\mathbf{p})$. Moreover, the lines connecting opposite vertices pass through the procedure line at the $\left(1, \frac{1}{3}, 0\right)$ normalized outcome, which is the midpoint of the procedure line (because of the $2 \frac{1 / 3}{1+(1 / 3)}=\frac{1}{2}$ distortion from the projection).

As with $\mathrm{AV}$, the $\mathrm{CV}$ hull is not affected by $\mathbf{C}^{3}$ components; however, $\mathrm{CV}$ values change with Basic components. The polyhedron has a regular shape for the kernel, and the Reversal terms alter the symmetry. Thus, again, a profile's Reversal symmetry is of particular interest in understanding the CV (and all multiple rules). All of this is captured in the following theorem; the proof is a simple computation. 
Theorem 6 For the three candidates $X, Y, Z$, the following are adjustments to the $C V$ polyhedron vertices relative to the the $\frac{1}{2}(3,1,0)$ tally.

a. On any profile or profile component, the diagonal points for relative adjustments differ by a multiple of - 1; e.g., $(L(A), M(B), H(C))=-(H(A), M(B), L(C))$.

b. The $\mathbf{C}^{3}$ adjustments are $H(X)=M(Y)=L(Z)=0$.

c. The $\mathbf{K}$ adjustments are $H(X)=2, M(Y)=0, L(Z)=-2$.

d. The $\mathbf{B}_{X}$ adjustments are $H(X)=1, M(Y)=-1.5$, and $L(Z)=0.5($ so $L(X)=$ $-1, M(Y)=1.5$, and $H(Z)=-0.5)$ and $M(X)=0, L(Y)=0.5, H(Z)=$ -0.5 .

e. $\quad$ The $\mathbf{R}_{X}$ adjustments are $H(X)=-1, L(Y)=-0.5, M(Z)=1.5$ and $M(X)=$ $0, L(Y)=-0.5, H(Z)=0.5$.

To illustrate with Fig. 4 profiles, Fig. 4 a outcome is a symmetric figure where the six vertices of the $\mathrm{CV}$ polyhedron are the six permutations of $(3,2,1)$.

The CV polyhedron for Fig. $4 \mathrm{~b}$ is more complex. With the plurality and antiplurality tallies of $(12,9,3)$ and $(20,17,11)$, the $\frac{1}{2}(3,1,0)$ tally, which is the center point for the $\mathrm{CV}$ polyhedron, is $\left(22,17 \frac{1}{2}, 8 \frac{1}{2}\right)$. In computing the adjustments for vertices in diametrically opposite pairs by using Theorem 6 , the $4 \mathbf{K}$ adjustments are immediate. For the $3 \mathbf{B}_{A}$ term, use part $d$ with $X=A$, and for the $2 \mathbf{B}_{B}$ term, let $X=B$. This leads to the computation $(H(A), M(B), L(C))=4(2,0,-2)+$ $3(1,-1.5,0.5)+2(-0.5,0,0.5)=(10,-4.5,-5.5)$, which means from part $a$ that $(L(A), M(B), H(C))=(-10,4.5,5.5)$. Each of these terms is added to the $\left(22,17 \frac{1}{2}, 8 \frac{1}{2}\right)$ center point to obtain two of the $\mathrm{CV}$ polyhedron vertices.

Using a similar computation,

$$
\begin{aligned}
(H(A), L(B), M(C)) & =4(2,-2,0)+3(1,0.5,-1.5)+2(-0.5,-1,1.5) \\
& =(10,-8.5,-1.5),
\end{aligned}
$$

which means that the $(L(A), H(B), M(C))$ increment is $(-10,8.5,1.5)$. The adjustment for the remaining pair is

$$
\begin{aligned}
(M(A), H(B), L(C)) & =4(0,2,-2)+3(0,-0.5,0.5)+2(-1.5,1,0.5) \\
& =(-3,8.5,-5.5),
\end{aligned}
$$

so the $(M(A), L(B), H(C))$ adjustment is $(3,-8.5,5.5)$. As this example demonstrates, the significant difference between the AV box and CV polyhedron is caused by the Basic terms.

With Fig. 4c profile, the $\mathbf{C}^{3}$ term does not effect the vertices. Thus, only the $\mathbf{R}_{A}$ term changes the $\mathrm{CV}$ polyhedron. The adjustments to Fig. $4 \mathrm{~b} \mathrm{CV}$ polyhedron follow:

$$
\begin{aligned}
& (H(A), M(B), L(C))=-(L(A), M(B), H(C)=(-1,1.5,-0.5) \\
& (H(A), L(B), M(C))=-(L(A) H(B), M(C))=(-1,-0.5,1.5) \\
& (M(A), H(B), L(C))=-(M(A), L(B), H(C))=(0,0.5,-0.5)
\end{aligned}
$$




\subsubsection{Who can beat whom?}

Again, by use of the geometry, we can find a simple rule to determine who could beat whom with a $\mathrm{CV}$ election. The rule involves the following condition, which is more liberal than the one in Definition 4.

Definition 5 Candidate $X$ is "weakly in the mix" with candidate $Y$ if the number of voters who do not have $X$ bottom ranked plus the number who have her top-ranked is more than the number of voters who have $Y$ top-ranked.

Theorem 7 For a given three-candidate profile, if $X$ is weakly in the mix with $Y$, then there exists a sincere $C V$ election outcome where $X$ beats $Y$. However, if $X$ is not weakly in the mix with $Y$, then no $C V$ outcome can have $X$ ranked above $Y$.

The proof follows from the geometry: If $X$ is weakly in the mix with $Y$, then $H(X)>L(Y)$. Thus, there are outcomes in the CV hull where $X$ beats $Y$. However, if $X$ is not weakly in the mix, then $H(X)<L(Y)$, so no CV outcome can rank $X$ over $Y$.

The difference between $X$ being in the mix with $Y$ and "weakly in the mix" is that $X$ 's first place rankings are double counted. This difference identifies the profiles with $\mathrm{CV}$ outcomes that can never arise with the AV; these profiles have a candidate $X$ who is not in the mix with $Y$, but who is weakly in the mix with $Y$. As an example, consider the 46 voter profile where

\begin{tabular}{ccc} 
Candidate & First place & Second place \\
\hline$A$ & 22 & 15 \\
$B$ & 20 & 16 \\
$C$ & 4 & 15
\end{tabular}

Only 19 voters have $C$ ranked above the bottom. As 19 is smaller than the number of first place votes for $A$ or $B, C$ is not in the mix with either candidate, so $C$ is bottom ranked in all $\mathrm{AV}$ rankings (Theorem 4 ). On the other hand, the number of voters who do not have $C$ bottom ranked, 19, plus the number who have her top ranked, 4, is 23, which exceeds the number of first place rankings for either other candidate. This means that $C$ is weakly in the mix with both $A$ and $B$, so there are $C V$ rankings where $C$ beats $A$, and other $\mathrm{CV}$ rankings where $C$ beats $B$ (Theorem 7).

A subtle geometric consequence of the $\mathrm{CV}$ polyhedron not being a rectangular box with edges parallel to each candidate's axis (as with AV) is that this profile does not have a $\mathrm{CV}$ outcome where $C$ is the winner. If, for instance, $C$ beats $A$, then $C$ has her highest possible CV tally of 23 to beat $A$ 's lowest tally of 22 . As the sum of the tallies is $2 \times 46=92$, it follows that $B$ tally is $92-[23+22]=47$; thus $B$ is the overwhelming first place $C V$ winner. Similarly, if $C$ beats $B, A$ will be the overwhelming CV winner.

The geometry identifies a condition to determine when $C$ can be a $\mathrm{CV}$ winner; it is that $H(C)>L(A), M(B)$ or $H(C)>M(A), L(B)$. Restated in words, if "twice the number of voters who do not have $X$ bottom ranked plus the number having her top-ranked exceeds the total number of voters plus the number of first place votes for either other candidate, then $X$ is top ranked in some $C V$ rankings." (This statement reflects the expression $H(X)=P_{X}+A_{X}>P_{Y}+\left(n-A_{X}\right)=M(Y) \geq L(Y)$.) 


\subsubsection{Completing the CV polyhedron}

The above describes all possible tallies that can occur should all voters select only between $(2,0,0)$ and $(1,1,0)$. To complete the story, we should compute the portion of the CV polyhedron coming from the $(1,0,0)$ and the $(1,1,0)$ options, which would be the AV box centered about the Borda tally. When tallies are normalized, however, this box adds nothing new to $\mathcal{T} H_{\mathrm{CV}}(\mathbf{p})$. The final option of selecting between $(2,0,0)$ and $(1,0,0)$ creates a geometric object defined by six vertices symmetrically positioned about the $\frac{1}{2}[(2,0,0)+(1,0,0)]=\left(\frac{3}{2}, 0,0\right)$ tally. Again, normalizing the tallies adds nothing new. The full $\mathrm{CV}$ polyhedron is the convex hull of these three geometric objects.

\section{The hulls and polyhedra for other methods}

I found the $\mathcal{T} \mathcal{H}_{\mathrm{CV}}(\mathbf{p})=\mathcal{T} \mathcal{H}_{\mathrm{DPV}}(\mathbf{p})$ expression to be surprising because the extra options DPV offers each voter make it reasonable to suspect that, in general, $\mathcal{T H}_{\mathrm{CV}}(\mathbf{p})$ is a proper subset of $\mathcal{T} \mathcal{H}_{\mathrm{DPV}}(\mathbf{p})$. To see why $\mathcal{T} \mathcal{H}_{\mathrm{CV}}(\mathbf{p}) \subset \mathcal{T} \mathcal{H}_{\mathrm{DPV}}(\mathbf{p})$, notice that if each voter is given $q$ points, then the admissible positional rules are $(q-s, s, 0)$ for any $0 \leq s \leq \frac{q}{2}$. But the extremes of $(q, 0,0)$ and $\left(\frac{q}{2}, \frac{q}{2}, 0\right)$ are equivalent to the CV's $(2,0,0)$ and $(1,1,0)$, so it follows that $\mathcal{T H}_{\mathrm{CV}}(\mathbf{p}) \subset \mathcal{T} \mathcal{H}_{\mathrm{DPV}}(\mathbf{p})$. With the scaling equivalence, we can assume that $q=2$ and $0 \leq s \leq 1$.

\subsection{The DPV and RV hulls}

To indicate why the $\mathcal{T} \mathcal{H}_{\mathrm{DPV}}(\mathbf{p})$ hull (but not the tallies) agrees with $\mathcal{T} \mathcal{H}_{\mathrm{CV}}(\mathbf{p})$, consider Eq. 6 profile. The seven CV voters with $A \succ C \succ B$ preferences permit tallies of $14-x$ to $A$ and $x$ to $C$. With DPV, the $i$ th voter gives $2-s_{i}$ points to $A$ and $s_{i}$ points to $C$. The total number of points assigned to $A$ and $C$ is, respectively, $14-\sum_{j=1}^{7} s_{j}$ and $\sum_{j=1}^{7} s_{j}$, where $0 \leq \sum_{j=1}^{7} s_{j} \leq 7$. Thus, the only difference in Eq. 6 is that the $x, y, z, u$ variables range over the continuum of values in their specified range rather than just the integer choices. As the same analysis holds for Eq. 16, we have that $\mathcal{T} \mathcal{H}_{\mathrm{CV}}(\mathbf{p})=\mathcal{T} \mathcal{H}_{\mathrm{DPV}}(\mathbf{p})$, which completes the proof.

One might expect a similar argument to show that $\mathcal{T H}_{\mathrm{RV}}(\mathbf{p})=\mathcal{T} \mathcal{H}_{\mathrm{AV}}(\mathbf{p})$, but this is not the case. For a simple proof, notice that an admissible RV option is where all voters use $\mathrm{CV}$. Thus $\mathcal{T} \mathcal{H}_{\mathrm{CV}}(\mathbf{p}) \subset \mathcal{T} \mathcal{H}_{\mathrm{RV}}(\mathbf{p})$. But, in general $\mathcal{T H}_{\mathrm{AV}}(\mathbf{p})$ is a proper $\mathcal{T} \mathcal{H}_{\mathrm{CV}}(\mathbf{p})$ subset, so $\mathcal{T} \mathcal{H}_{\mathrm{RV}}(\mathbf{p})$ includes and, in general, is larger than $\mathcal{T} \mathcal{H}_{\mathrm{AV}}(\mathbf{p})$.

To find $\mathcal{T} \mathcal{H}_{\mathrm{RV}}(\mathbf{p})$ use the same approach: $\mathcal{T} \mathcal{H}_{\mathrm{RV}}(\mathbf{p})$ is the convex hull of its extreme points. The almost unrestrained array of options provided the voters means that the extreme points include tallies where all voters with a certain preference vote only for their top ranked candidate giving her the highest number of points and all other voters give zero to all candidates, or all voters with a certain preference give the highest points to their top two candidates and all other voters give zero to their candidates. [As true with all hulls, the extreme situations need not occur in actual elections. 
Nevertheless, because so many options are provided to each voter, in general this computed $\mathcal{T} \mathcal{H}_{\mathrm{RV}}(\mathbf{p})$ is closely packed with $\mathrm{RV}$ tallies.]

As a result, if each candidate is top-ranked by at least one voter, then $\mathcal{T} \mathcal{H}_{\mathrm{RV}}(\mathbf{p})$ is the total simplex; anything and any normalized tally can happen! More generally, $\mathcal{T H}_{\mathrm{RV}}(\mathbf{p})$ is the convex region connecting all vertices where some voter has that candidate top-ranked, and all midpoints on the edge connecting vertices where some voter has the two candidates in the top two ranks.

To extend this assertion to any number of candidates, include vertices to account for when a candidate is not first or second ranked; e.g., all center points of a triangle where some voter has all three candidates in the top three places, etc. In general, $\mathcal{T} \mathcal{H}_{\mathrm{RV}}(\mathbf{p})$ includes everything, including $\mathcal{T} \mathcal{H}_{\mathrm{DPV}}(\mathbf{p})$ as a proper subset. else. The associated RV condition for admissible rankings is extremely liberal; if a candidate is top-ranked by just one voter, she can be the RV winner.

\subsection{In general}

The hulls and polyhedra for other multiple methods are similarly constructed: determine the convex hull by the rule's extreme points. For multiple rules where one-way of voting subtracts points from the voter's top choice, such as where a voter chooses between, say $(5,1,0)$ and $(4,3,0)$, guidance comes from the $\mathrm{CV}$ construction. For rules where voting for a lower ranked candidate does not influence the number of points assigned to a top-ranked candidate, the geometry mimics the AV analysis; e.g, this is true for truncated voting or if a voter is to select between $(1,0,0)$ and $\left(1, \frac{1}{3}, 0\right)$.

As multiple rules rely on positional rules, the $\mathbf{C}^{3}$ profile component does not affect them. In general, then, no relationship exists between the multiple rule's ranking and the majority vote's ranking over pairs. (An exception is if the Borda Count is used.) For all multiple rules, the differing shapes of its hull and polyhedron are determined by the Reversal components of a profile. Each face, which is determined by two different positional rules, is symmetrically situated about the "averaged" positional rule's tally. Thus, a simpler way to compute vertices for the polyhedron is to find how the Reversal, Basic, and Kernel components create adjustments to the central positional rule's tally. As illustrated by the above theorems, these adjustment terms are found by computing the behavior of the diagonal vertices on Fig. 3 type components.

\section{Designer's choice}

Because the hulls for AV, CV, DPV, and several other multiple rules are completely determined by the plurality and antiplurality tallies, it is possible to start with a rough polyhedral design of the hull and then determine whether the hull is supported by some profile. To develop the approach, first notice that a candidate's AP tally includes her plurality tally, so $P_{X} \leq A_{X}$. But as each voter has twice as many AP votes to distribute, the normalized values satisfy $\frac{1}{2} \frac{P_{X}}{n} \leq \frac{A_{X}}{2 n}$. Finally, a candidate can receive, 
at most, half of the AP votes, so her normalized AP tally is bounded above by $\frac{1}{2}$. In summary,

$$
\frac{1}{2} \frac{P_{X}}{n} \leq \frac{A_{X}}{2 n} \leq \frac{1}{2} .
$$

\subsection{Finding a profile}

Surprisingly, as the next theorem (proof in the Appendix) asserts, Eq. 18 characterizes whether $\mathrm{P}$ and AP tallies can be supported by a profile. The assertion in Theorem 8 about the role of Condorcet symmetries is illustrated in Eq. 13.

Theorem 8 Let $\mathbf{P}_{j}=\left(p_{A}^{j}, p_{B}^{j}, p_{C}^{j}\right), j=1,2$, be any two points in the simplex where each component is a fraction. Suppose for each $X$ that

$$
\frac{1}{2} p_{X}^{1} \leq p_{X}^{2} \leq \frac{1}{2}
$$

There exist profiles so that $\mathbf{P}_{1}$ and $\mathbf{P}_{2}$ are, respectively, their normalized $P$ and $A P$ outcomes. These profiles differ only in that any profile can be multiplied by a positive integer and that scalar versions may differ by multiples of $\mathbf{C}^{3}$.

Profiles exist, but how are they found? To illustrate, let $\mathbf{P}_{1}=\mathbf{P}_{2}=\left(\frac{1}{2}, \frac{1}{6}, \frac{1}{3}\right)$. (As the points agree, the associated procedure line collapses to this common point.) To find a profile, multiply $\mathbf{P}_{1}$ by the common denominator of 6 to obtain the $P$ integer values of $(3,1,2)$. To find AP integer choices, from $\mathbf{P}_{2}$, use the fact that the sum of AP tallies is twice that of the P tallies. So, multiply $\mathbf{P}_{2}$ by twice this value, or 12 , to obtain $(6,2,4)$. Thus, $\operatorname{STP}=(6,2,4)-(3,1,2)=(3,1,2)$. The $P$ and STP values identify, respectively, how many first and second place votes each candidate receives. Armed with this information, finding a profile involves only simple algebra. In this case, a supporting profile has one voter preferring $A \succ B \succ C$, one $B \succ A \succ C$, two $C \succ A \succ B$ and two $A \succ C \succ B$.

As Theorem 8 asserts, this profile defines a class of profiles. Namely, any positive integer $m$ multiple of this profile defines a related profile with the same normalized A and AP tallies:

\begin{tabular}{cc|cc} 
Number & Ranking & Number & Ranking \\
\hline$m$ & $A \succ B \succ C$ & $2 m$ & $C \succ A \succ B$ \\
$2 m$ & $A \succ C \succ B$ & $m$ & $B \succ A \succ C$
\end{tabular}

These profiles cannot differ by $\mathbf{C}^{3}$; the reason is that adding any $\mathbf{C}^{3}$ multiple to Eq. 20 results in negative components.

For an example where $\mathbf{C}^{3}$ does play a role, let $\mathbf{P}_{1}=\left(\frac{4}{13}, \frac{3}{13}, \frac{6}{13}\right)$ and $\mathbf{P}_{2}=$ $\left(\frac{8}{26}, \frac{8}{26}, \frac{5}{13}\right)$. As $\mathbf{P}_{1}, \mathbf{P}_{2}$ satisfy Eq. 19, it follows from Theorem 8 that supporting profiles exist. To find them, multiply $\mathbf{P}_{1}$ by 13 and $\mathbf{P}_{2}$ by 26 to obtain that $P=(4,3,6)$ 
and $S P T=(8,8,10)-(4,3,6)=(4,5,4)$. There are four differing 13 -voter profiles:

\begin{tabular}{cc|cc} 
Number & Ranking & Number & Ranking \\
\hline$x$ & $A \succ B \succ C$ & $5-x$ & $C \succ B \succ A$ \\
$4-x$ & $A \succ C \succ B$ & $x$ & $B \succ C \succ A$ \\
$1+x$ & $C \succ A \succ B$ & $3-x$ & $B \succ A \succ C$
\end{tabular}

where the $0 \leq x \leq 3$ admissible $x$ values define the different $\mathbf{C}^{3}$ multiples. A property associated with these $\mathbf{C}^{3}$ multiples is that at least one supporting profile has no voters with a certain ranking; this always happens. So, to find a supporting profile, start by assigning zero voters to some ranking; e.g., as $B$ has only 3 first place votes in this example, I assigned zero voters to $B \succ C \succ A$ and three to $B \succ A \succ C$. The rest of the base profile (with $x=0$ ) followed immediately.

\subsection{Finding polyhedra}

After selecting any two points $\mathbf{P}_{1}$ and $\mathbf{P}_{2}$ that satisfy Eq. 19, the next task is to find a multiple rule's outcomes for the associated profiles. To illustrate with Eq. 20 profile, notice that $A$ is never bottom ranked and $C$ is not bottom ranked by $4 m$, or two-thirds of them; thus $A$ and $C$ are "in the mix" with all other candidates; i.e., each is top ranked with certain $\mathrm{AV}$ outcomes. On the other hand, $B$ is not bottom ranked by $2 m$ of the $6 m$ voters, so she is not in the mix with either $A$ or $C$; the best she can do is to AV tie with $C$ for bottom place. $B$, however, is weakly in the mix with $C$ (but not $A$ ), so certain behavioral assumptions allow $B$ to be $\mathrm{CV}$ second ranked. As each candidate is top ranked by some voter, expect anything to happen with RV; e.g., even sincere RV outcomes can have $B$ top ranked.

This analysis requires finding a supporting profile for the selected $\mathbf{P}_{1}$ and $\mathbf{P}_{2}$. Fortunately, as developed below, it is not necessary to construct a profile to discover these different multiple rule behaviors; answers follow directly from the $\mathbf{P}_{1}$ and $\mathbf{P}_{2}$ components. So, after selecting any two points in the simplex that satisfy Eq. 19, we can immediately determine the kinds of outcomes and properties associated with each of the different multiple rules.

\subsubsection{Procedure line}

The straight line connecting any choice of $\mathbf{P}_{1}$ and $\mathbf{P}_{2}$ that satisfies Eq. 19 is the procedure line for a class of profiles. Consequently, to find all possible positional election behaviors, just experiment with how a straight line can be positioned in the simplex! Among sample results, because a straight line crosses at most seven ranking regions, it follows that a profile can generate, at most, seven different positional outcomes. As another result, if the outcome for some positional method is a complete tie (which means that the procedure line crosses the simplex's midpoint), then (a) all outcomes are ties, (b) the outcome is the P or AP outcome and all other rankings agree, or (c) all other positional outcomes come from two other election rankings that reverse each other. 


\subsubsection{AV hull}

For any choice of $\mathbf{P}_{1}$ and $\mathbf{P}_{2}$ that satisfy Eq. 19 points, if the points also satisfy $2 p_{X}^{2}>p_{Y}^{1}$ (so " $X$ is in the mix with $Y$ "), then the resulting AV hull has outcomes where $X$ beats $Y$. To illustrate, if twice the value each $\mathbf{P}_{2}$ component is larger than any of the $\mathbf{P}_{1}$ components, then all 13 rankings are admissible $\mathrm{AV}$ rankings. With the earlier $\mathbf{P}_{1}=\left(\frac{4}{13}, \frac{3}{13}, \frac{6}{13}\right)$ and $\mathbf{P}_{2}=\left(\frac{8}{26}, \frac{8}{26}, \frac{5}{13}\right)$, as each component of $2 \mathbf{P}_{2}=\left(\frac{8}{13}, \frac{8}{13}, \frac{10}{13}\right)$ is larger than any component of $\mathbf{P}_{1}$, all 13 rankings are admissible AV outcomes. With $\mathbf{P}_{1}=\mathbf{P}_{2}=\left(\frac{1}{2}, \frac{1}{6}, \frac{1}{3}\right)$, as $2 \mathbf{P}_{2}=\left(1, \frac{1}{3}, \frac{2}{3}\right)$, it follows by a comparison of these components that the AV rankings include any $\{A, C\}$ ranking where $A \succ B$ and $C \succeq B$.

Other results can be obtained by using algebra with Eq. 19. For instance, it now is easy to determine which $\mathbf{P}_{1}, \mathbf{P}_{2}$ points, hence which profiles, have a specified number of strict $\mathrm{AV}$ outcomes. For instance, finding all profiles where the AV rankings have a single strict $\mathrm{AV}$ ranking, say $A \succ B \succ C$, is equivalent to finding all $\mathbf{P}_{1}, \mathbf{P}_{2}$ that satisfy $p_{A}^{1} \geq 2 p_{B}^{2} \geq p_{B}^{1} \geq 2 p_{C}^{2} \geq p_{C}^{1}$, which identifies a region near the $A$ vertex. Finally, to construct the associated AV box and hull for the class of profiles that are defined by the selected $\mathbf{P}_{1}, \mathbf{P}_{2}$, just use the eight extreme points created by $\mathbf{P}_{1}$ and $2 \mathbf{P}_{2}$.

\subsubsection{CV hull}

Finding potential CV hulls is immediate. Select any $\mathbf{P}_{1}, \mathbf{P}_{2}$ that satisfy Eq. 19. Determining the associated CV hull mimics the construction in Fig. 5b. Namely, first draw three solid line where each is parallel to an edge; as these lines represent the $L(X)$ tallies, for $X$, designate the value as $X_{L}$. As $2\left(A_{L}, B_{L}, C_{L}\right)=\mathbf{P}_{1}$, we have that $A_{L}=\frac{1}{2} p_{A}^{1}, B_{L}=\frac{1}{2} p_{B}^{1}, C_{L}=\frac{1}{2} p_{C}^{1}$. Next draw three dashed lines parallel to the edges and "above" (i.e., closer to the vertex) the solid lines; as these values represent $H(X)$ values, for $X$, denote it as $X_{H}$. As $\mathbf{P}_{2}=\left(A_{H}-A_{L}, B_{H}-B_{L}, C_{H}-C_{L}\right)$, we have that $A_{H}=p_{A}^{2}+\frac{1}{2} p_{A}^{1}, B_{H}=p_{B}^{2}+\frac{1}{2} p_{B}^{1}, C_{H}=p_{C}^{2}+\frac{1}{2} p_{C}^{1}$. The $\mathbf{P}_{1}, \mathbf{P}_{2}$ points satisfy Eq. 19 inequalities, so the resulting hull is supported by a class of profiles. (Equation 19 inequalities assume the $X_{L} \leq X_{H}-X_{L} \leq \frac{1}{2}$, or $2 X_{L} \leq X_{H} \leq \frac{1}{2}+X_{L}$ forms.)

For selected points $\mathbf{P}_{1}, \mathbf{P}_{2}$ that satisfy Eq. 19 and $2 p_{X}^{2}+p_{X}^{1}>p_{Y}^{1}$, the resulting $\mathrm{CV}$ hull has outcomes where $X$ beats $Y$. Again algebra can be used to identify which profiles allow how many and what kinds of CV rankings. To CV hull is constructed by using the $\mathbf{P}_{1}, \mathbf{P}_{2}$ values.

\section{Consequences}

What makes it difficult to systematically analyze the behavioral assumptions associated with each multiple rule is that, in general (Sect. 2), an election tally may be supported by many different election scenarios. As no single explanation suffices, all should be explored. To identify all possibilities, label the extreme points in the appropriate hull; e.g., for the CV hull, the vertices are of the $(L(A), H(B), M(C))$ form. These labels make it possible to "eyeball" the relative locations of the labeled points with respect to a specified outcome and determine all possible supporting voter 
behaviors. With Fig. 2c, for instance, because the Borda tally is at the center of the AV hull, there are several different symmetry scenarios where an AV tally will agree with the Borda outcome. Similarly, several symmetric scenarios have the CV tally agreeing with the $\left(1, \frac{1}{3}, 0\right)$ tally.

Recall the question raised in the introduction as to whether there exist profiles that admit opposite DPV rankings; if so, can they be characterized? By using the profile decomposition to construct these hulls, we now know that for any of the four multiple rules, any profile with a large Reversal component, relative to the Basic component, has this property.

To illustrate the geometry of the hulls with respect to the motivating Guinier CV example, in practical settings polls can be used to estimate the election's $\mathbf{P}_{1}, \mathbf{P}_{2}$ values; from these values, a CV hull can be constructed. Whatever strategy a minority adopts to try to elect candidate $C$, it provides an estimate for $C$ 's lowest possible tally. To determine everything else that can possibly happen, draw a horizontal line in the simplex with the ensured $C$ tally; all other possible outcomes, with their associated behaviors, are in the part of the CV hull above this line. For a theoretical analysis, use the Sect. 6 material to construct all possible CV hulls and explore the different behavioral themes.

The size and number of tallies in these hulls must make one skeptical of any assertion claiming that a specified mutiple rule will have a particular kind of outcome. In the preceding paragraphs, for instance, I showed that by assuming a highly symmetrical behavior over all types of voters, the AV outcome tends to agree with the Borda outcome. The mathematical assertion is correct, but the behavioral assumption is highly questionable. What we need is more information about actual behavior, such as Regenwetter et al. (2007) statistical results showing that when using AV, many voters tend to vote for only one candidate. If this result holds in general, the associated lack of symmetry would seriously question any assertion that the AV outcome tends to agree with, say, the Borda outcome.

An attraction of multiple rules, which often is used to promote them, is how they offer a voter several ways to reflect personal views of the candidates. But, as true in all societal considerations, a balance must be found between individual flexibility and the societal good; e.g., while I personally enjoy driving my car at high speeds, societal realities require me to respect restraining traffic laws. Similarly, the geometric approach developed here helps us to better determine the hidden societal costs that are associated with using different multiple rules. Such changes include targeted campaigning, strategic voting, and even the ability of those with harmful intent to disrupt the outcome.

Indeed, discussions about multiple rules tend to emphasize what can happen with positive behavioral assumptions, but negative behavior is a contemporary reality that must be recognized and explored. Here, the size and shape of the hull identifies what can happen; e.g., rules with larger hulls, such as RV, are more suspectible to such traits, whether positive, negative, or accidental. For instance, if voters can successfully alter outcomes (without changing the profile) through strategic voting, then the indifference lines, or lines reflecting tied outcomes, must pass through the rule's hull. Thus, a crude measure of this ability to alter the societal sincere outcome is the relative lengths of these indifference lines within the hulls. A first, crude measure of the relative suscep- 
tibility of the multiple rules to strategic and negative behavior, then, is given by the Theorem 2 inclusions.

This measure indicates that RV is, by far, the most susceptible to strategic voting and other kinds of negative activities. But with the ease of being strongly strategic with $\mathrm{RV}$, such a comment must be expected. Historical support for this assertion comes from the fact that RV includes, as a special case, those earlier versions of the scoring rules used for figure skating that were discredited because of the persistent and serious levels of manipulation.

Open Access This article is distributed under the terms of the Creative Commons Attribution Noncommercial License which permits any noncommercial use, distribution, and reproduction in any medium, provided the original author(s) and source are credited.

\section{Appendix}

Prof of Theorem 8 Suppose $\mathbf{P}_{1}, \mathbf{P}_{2}$ satisfy Eq. 19 and have rational components. We need to show that the class of supporting profiles is non-empty and that if $\mathbf{p}_{1}$ and $\mathbf{p}_{2}$ are two different such profiles with the same number of voters, then $\mathbf{p}_{2}-\mathbf{p}_{1}$ is a multiple of $\mathbf{C}^{3}$, or if $\mathbf{p}_{1}$ and $\mathbf{p}_{2}$ do not have the same number of voters, then there exist positive integers $d_{j}$ so that $d_{2} \mathbf{p}_{2}-d_{1} \mathbf{p}_{1}$ is a multiple of $\mathbf{C}^{3}$. As the scalar assertion is clear, only the $\mathbf{C}^{3}$ claim needs to be verified.

Using Eq. 14 labeling of preferences, we have $p_{A}^{1}=p_{1}+p_{2}=P_{A}, p_{B}^{1}$ $=p_{5}+p_{6}=P_{B}, p_{C}^{1}=p_{3}+p_{4}=P_{C} ; A_{A}=2 p_{A}^{2}=P_{A}+p_{3}+p_{6}, A_{B}=$ $2 p_{A}^{2}=P_{B}+p_{1}+p_{4}, A_{C}=2 p_{C}^{2}=P_{C}+p_{2}+p_{5}$. The associated matrix representation is

$$
\left(\begin{array}{llllll}
1 & 1 & 0 & 0 & 0 & 0 \\
0 & 0 & 0 & 0 & 1 & 1 \\
0 & 0 & 1 & 1 & 0 & 0 \\
1 & 1 & 1 & 0 & 0 & 1 \\
1 & 0 & 0 & 1 & 1 & 1 \\
0 & 1 & 1 & 1 & 1 & 0
\end{array}\right)\left(\begin{array}{l}
p_{1} \\
p_{2} \\
p_{3} \\
p_{4} \\
p_{5} \\
p_{6}
\end{array}\right)=\left(\begin{array}{c}
p_{A}^{1} \\
p_{B}^{1} \\
p_{C}^{1} \\
2 p_{A}^{2} \\
2 p_{B}^{2} \\
2 p_{C}^{2}
\end{array}\right)
$$

For a given $\mathbf{P}^{1}, \mathbf{P}^{2}$, all supporting profiles are obtained from this matrix representation. But the matrix has rank 5 , so it has a kernel. As $\mathbf{C}^{3}=(1,-1,1,-1,1,-1)$ is orthogonal to each row, the kernel is the linear space spanned by $\mathbf{C}^{3}$. Thus, as asserted, all solutions (i.e., all supporting profiles) with a fixed number of voters differ by multiples of $\mathbf{C}^{3}$.

The matrix expression shows that if Eq. 19 is not satisfied, then some $p_{j}$ terms must be negative; e.g., if $2 p_{C}^{2}<p_{C}^{1}$, then from the third and sixth row, or $2 p_{C}^{2}=$ $p_{C}^{1}+p_{2}+p_{5}$, we have that $p_{2}+p_{5}<0$. Somewhat of a surprise, Eq. 19 constraints affect only the number of voters in a profile by imposing a lower bound on the admissible $\mathbf{K}$ multiples. To illustrate by finding the profile decomposition $\mathbf{p}=k \mathbf{K}+a_{B} \mathbf{B}_{A}+b_{B} \mathbf{B}_{B}+a_{R} \mathbf{R}_{A}+b_{R} \mathbf{R}_{B}$ for $P=(6,5,4)$ and AP $=(6,9,15)$, 
as the sum of $P$ coordinates is 15 , there are 15 voters and $k=15 / 6$. To remove the $\mathbf{K}$ influence from the tallies, subtract 5 and 10, respectively, from each $P$ and AP component to obtain the reduced values of $(1,0,-1)$ and $(-4,-1,5)$.

The reduced Borda outcome - the Borda tally minus the $\mathbf{K}$ effect - is the average, or $\left(-\frac{3}{2},-\frac{1}{2}, 2\right)$. This reduced Borda outcome is determined exclusively by the Basic terms, so, according to the $\mathbf{B}_{X}$ form (e.g., Fig. 3), the $a_{B}, b_{B}$ coefficients are given by

$$
2 a_{B}-b_{B}=-\frac{3}{2}, \quad 2 b_{B}-a_{B}=-\frac{1}{2}, \quad-a_{B}-b_{B}=2 .
$$

The $a_{R}, b_{R}$ values are determined by the difference between the reduced $P$ and Borda tallies, or $(1,0,-1)-\left(-\frac{3}{2},-\frac{1}{2}, 2\right)=\left(\frac{5}{2}, \frac{1}{2},-3\right)$. Using the $\mathbf{R}_{X}$ representations with $s=0$, the equations are

$$
2 a_{R}-b_{R}=\frac{5}{2}, \quad 2 b_{R}-a_{R}=\frac{1}{2}, \quad-a_{R}-b_{R}=-3 .
$$

As the sums of coefficients for a reduced Borda tally and for the differences between the reduced plurality and Borda tallies always equal zero, whatever the values, the respective form of Eqs. 22 and 23 (but with the new values on the right-hand side) always have an unique solution. Thus the Eq. 19 effect is manifested only through the $k \mathbf{K}$ term.

To see how this happens, keep the above $P=(6,5,4)$ but now use AP $=$ $(4,11,15)$, which is a 15 voter example that violates Eq. 19. The reduced $\mathrm{P}$ and AP values are $(1,0,-1)$ and $(-6,1,5)$, from which corresponding $a_{B}, b_{B}, a_{R}, b_{R}$ values can be computed. However, working backwards, the appropriate $k$ coefficient for $k \mathbf{K}$ must correspond to $P$ and AP values of $(2 k+1,2 k, 2 k-1)$ and $(4 k-6,4 k+1,4 k+5)$. The requirement that $P_{X} \leq A_{X}$ means that $2 k+1 \leq 4 k-6$, or that $6 k$, the number of voters, must be at least 21 , rather than 15 .

\section{References}

Arrow KJ (1963) Social choice and individual values, 2nd edn. Wiley, New York

Brams S, Fishburn P (1978) Approval voting. Birkhausere, Boston

Brams S, Fishburn P, Merrill S et al (1988) The responsiveness of approval voting: comments on Saari and Van Newenhizen. Public Choice 59(2):121-131

Nurmi H (2002) Voting procedures under uncertainty. Springer, New York

Regenwetter M, Ho M-HR, Tsetlin I (2007) Sophisticated approval voting, ignorance priors, and plurality heuristics. Am Psychol Rev 114(4):994-1014

Saari DG (1992) Millions of election outcomes from a single profile. Soc Choice Welf 9:277-306

Saari DG (1995) Basic geometry of voting. Springer, New York

Saari DG (2001) Chaotic elections! American Mathematical Society, Providence

Saari DG (1999) Explaining all three-alternative voting outcomes. J Econ Theory 87:313-335

Saari DG (2008) Disposing dictators, demystifying voting paradoxes. Cambridge University Press, New York

Saari DG (2001) Analyzing a "nail-biting" election. Soc Choice Welf 18:415-430

Saari DG, Van Newenhizen J (1988) Is approval voting an “unmitigated evil?” Public Choice 59:133-147

Tabarrok A (2001) President Perot or fundamentals of voting theory illustrated with the 1992 election, or could Perot have won in 1992? Public Choice 106:275-297

Tabarrok A, Spector L (1999) Would the Borda Count have avoided the civil war? J Theor Polit 11:261-288 